\title{
Wafer-based aberration metrology for lithographic systems using overlay measurements on targets imaged from phase- shift gratings
}

\author{
Citation for published version (APA): \\ Haver, van, S., Coene, W. M. J. M., D'havé, K., Geypen, N., Adrichem, van, P., Winter, de, L., Janssen, A. J. E. \\ M., \& Cheng, S. (2014). Wafer-based aberration metrology for lithographic systems using overlay measurements \\ on targets imaged from phase-shift gratings. Applied Optics, 53(12), 2562-2582. \\ https://doi.org/10.1364/AO.53.002562
}

DOI:

10.1364/AO.53.002562

Document status and date:

Published: 01/01/2014

\section{Document Version:}

Publisher's PDF, also known as Version of Record (includes final page, issue and volume numbers)

\section{Please check the document version of this publication:}

- A submitted manuscript is the version of the article upon submission and before peer-review. There can be important differences between the submitted version and the official published version of record. People interested in the research are advised to contact the author for the final version of the publication, or visit the DOI to the publisher's website.

- The final author version and the galley proof are versions of the publication after peer review.

- The final published version features the final layout of the paper including the volume, issue and page numbers.

Link to publication

\footnotetext{
General rights

- You may freely distribute the URL identifying the publication in the public portal. follow below link for the End User Agreement:

www.tue.nl/taverne

\section{Take down policy}

If you believe that this document breaches copyright please contact us at:

openaccess@tue.nl

providing details and we will investigate your claim.
}

Copyright and moral rights for the publications made accessible in the public portal are retained by the authors and/or other copyright owners and it is a condition of accessing publications that users recognise and abide by the legal requirements associated with these rights.

- Users may download and print one copy of any publication from the public portal for the purpose of private study or research.

- You may not further distribute the material or use it for any profit-making activity or commercial gain

If the publication is distributed under the terms of Article $25 \mathrm{fa}$ of the Dutch Copyright Act, indicated by the "Taverne" license above, please 


\title{
Wafer-based aberration metrology for lithographic systems using overlay measurements on targets imaged from phase-shift gratings
}

\author{
Sven van Haver, ${ }^{1,2, \star}$ Wim M. J. Coene, ${ }^{1}$ Koen D'havé, ${ }^{3}$ Niels Geypen, ${ }^{1,4}$ \\ Paul van Adrichem, ${ }^{1}$ Laurens de Winter, ${ }^{1}$ Augustus J. E. M. Janssen, ${ }^{5}$ \\ and Shaunee Cheng $^{3}$ \\ ${ }^{1}$ ASML, Flight Forum 1900, 5657 EZ Eindhoven, The Netherlands \\ ${ }^{2}$ S[\&]T Experts Pool (STEP), Olof Palmestraat 14, 2616 LR Delft, The Netherlands \\ ${ }^{3}$ IMEC, Kapeldreef 75, B-3001 Leuven, Belgium \\ ${ }^{4}$ Sioux, LIME BV, Esp 405, 5633 AJ Eindhoven, The Netherlands \\ ${ }^{5}$ Department of Mathematics and Computer Science, Eindhoven University of Technology, \\ P.O. Box 513, 5600 MB Eindhoven, The Netherlands \\ ${ }^{*}$ Corresponding author: svenvanhaver@gmail.com
}

Received 10 January 2014; revised 28 February 2014; accepted 1 March 2014; posted 10 March 2014 (Doc. ID 204555); published 15 April 2014

\begin{abstract}
In this paper, a new methodology is presented to derive the aberration state of a lithographic projection system from wafer metrology data. For this purpose, new types of phase-shift gratings (PSGs) are introduced, with special features that give rise to a simple linear relation between the PSG image displacement and the phase aberration function of the imaging system. By using the PSGs as the top grating in a diffraction-based overlay stack, their displacement can be measured as an overlay error using a standard wafer metrology tool. In this way, the overlay error can be used as a measurand based on which the phase aberration function in the exit pupil of the lithographic system can be reconstructed. In practice, the overlay error is measured for a set of different PSG targets, after which this information serves as input to a least-squares optimization problem that, upon solving, provides estimates for the Zernike coefficients describing the aberration state of the lithographic system. In addition to a detailed method description, this paper also deals with the additional complications that arise when the method is implemented experimentally and this leads to a number of model refinements and a required calibration step. Finally, the overall performance of the method is assessed through a number of experiments in which the aberration state of the lithographic system is intentionally detuned and subsequently estimated by the new method. These experiments show a remarkably good agreement, with an error smaller than $5 \mathrm{~m} \lambda$, among the requested aberrations, the aberrations measured by the on-tool aberration sensor, and the results of the new wafer-based method. (C) 2014 Optical Society of America

OCIS codes: (120.0120) Instrumentation, measurement, and metrology; (220.3740) Lithography; (100.5070) Phase retrieval; (220.1010) Aberrations (global); (050.0050) Diffraction and gratings.

http://dx.doi.org/10.1364/AO.53.002562
\end{abstract}

$1559-128 \mathrm{X} / 14 / 122562-21 \$ 15.00 / 0$

(C) 2014 Optical Society of America

\section{Introduction}

In recent years, the specifications and tolerances for aberration control in the lithographic industry have reached a level at which the contributions from 
dynamic effects also form a significant fraction of the overall aberration budget of a lithographic system. In this context, an aberration is considered dynamic when its contribution may change during the complete exposure of a wafer. This includes, for example, lens heating, vibrational modes of lens elements due to the scanning motion of the reticle stage, or even refractive index variations of the immersion fluid caused by flow or bubbles. Although, modern day lithographic systems are all equipped with on-tool sensors to measure their aberrations, these sensors commonly operate in between wafer exposures and consequently are unable to pick up the dynamics of the mentioned effects. In the present work we aim at developing an alternative wafer-based aberration metrology method that can pick-up and study these effects.

In the semiconductor industry, lithography is applied to transfer a desired pattern from a reticle onto a light-sensitive layer on a wafer. If aberrations are present, these give rise to deformations of the transferred pattern, like lateral feature displacements and blurring effects, which in turn may lead to malfunctioning electronic circuits or chips. As a consequence, the tolerances for these effects are extremely tight. For example, current and future lithographic nodes have an overall feature displacement budget that is of the order of a few nanometers, and this includes, in addition to aberrations, contributions from other sources, such as wafer stage positioning errors and reticle defects. Effectively this means that, for state of the art lithographic processes, aberrations should be controlled down to the subnanometer regime and this implies that, for a lithographic system operating at a wavelength of $193 \mathrm{~nm}$, a phase measurement accuracy of the order of a few milliwaves is required. In the current work we therefore aim at a desired aberration measurement accuracy that is better than $2.5 \mathrm{~m} \lambda$.

Since aberration control in the semiconductor industry is mainly driven by the tight error tolerances for the features being printed, it is also true that aberrations are considered relevant only if they significantly alter the pattern being printed. Looking at the problem from this perspective, it seems natural to monitor the aberration state of a lithographic system based on information extracted from the wafer. However, quantitative wafer-based aberration analysis, down to the required accuracy, proves to be very challenging. Not only are the aberrationinduced changes very small, they are also strongly correlated with other feature shape changing effects, such as focusing and wafer processing. Because of these and other complications, wafer-based aberration metrology methods have never become an industry standard that is widely used. Nevertheless, we will show in this paper that the above-mentioned issues can be overcome and that a wafer-based method can be devised that is an excellent candidate for studying dynamic aberration effects.

The wafer-based aberration metrology method presented here is inspired by a paper by Nomura published in 2001 [1]. In this paper, Nomura introduced a special type of grating, based on which a method could be devised to distinguish between odd and even aberrations simply by measuring the aberration-induced image shift for a few of these gratings. The targets used by Nomura are of the phaseshift grating (PSG) type and basically consist of binary line-space gratings for which part of the light transmitting area is phase shifted by means of an etch into the grating substrate of the mask. The current work builds upon Nomura's pioneering ideas and further exploits the aberration information contained in the image position of a PSG.

By careful design of a PSG, and satisfying a number of constraints, a simple linear relation between the PSG image displacement and the phase aberration function of the imaging system can be established. This will be explained in detail in Section 2 and basically provides the possibility to obtain phase information, for a single point in the exit pupil of the imaging optics, by measuring the PSG image displacement. However, in order to determine the aberrations of an optical system, the phase distribution in the entire pupil is required. In the proposed method, we therefore use many $1 \mathrm{D}$ - and 2D-PSGs, having different grating pitches and rotation in the object plane, to effectively probe the phase distribution at different locations in the pupil. In this way, enough phase information can be collected to reconstruct the aberration function by means of solving a least-squares optimization problem, and the only task that remains is the accurate measurement of the lateral image displacements of all PSGs to serve as input.

Measuring relative displacements is a common task in the semiconductor industry. An electronic circuit is built up from many different layers that should be stacked on top of each other very accurately to assure a correctly functioning chip. Layer placement is monitored by dedicated wafer overlay metrology systems, which basically measure the relative displacement (known in the industry as the overlay error) between two functional layers on the wafer by comparing dedicated targets included in both layers. In the current work we exploit this existing overlay measurement technology by replacing the target in one layer by a PSG-based target to directly measure the aberration-induced PSG image shift relative to the target in the other (reference) layer.

By the end of Section 2 we will have introduced all basic concepts and measurement principles required to devise a PSG overlay (PSG-OVL) based aberration metrology method, and we will give a proof-ofprinciple in simulation to show its feasibility. From there on, the remainder of the paper is organized as follows. Section 3 deals with the additional complications that arise when the proposed method is implemented experimentally. There it will be shown that, although some of the distinguished experimental issues have the potential to significantly degrade the performance of the method, they can all be 
accounted for, or compensated for, through a number of model refinements and a calibration operation. In Section 4 the implementation of the method is discussed and experimental results are given for a number of aberration reconstruction examples. This will also illustrate the impact of the various model refinements and the calibration operation introduced in Section 3. Section 4 is concluded with an experiment in which the relative aberrations measured by the new wafer-based method and an on-tool aberration sensor (based on shearing interferometry [2]) are compared, showing both methods to agree to a level better than $5 \mathrm{~m} \lambda$. We will conclude with the overview of our work in Section $\underline{5}$, where we shall summarize and comment on the results obtained and where we shall give recommendations for further development of the method.

\section{PSG-OVL Aberration Metrology}

In this section, the basic concepts and measurement principles exploited in this paper are discussed. Based on a simplified image formation model and new types of PSGs, it is shown that a straightforward relation can be realized between the displacement of a PSG image and the aberrations of the imaging system. In addition, it is explained how standard wafer metrology tools can be exploited to measure this aberration-induced displacement for a tailored set of different PSGs, in this way collecting enough information to allow estimation of the aberration function of the optical system.

\section{A. Aerial Image and Two-Beam Interference}

The simplified image formation model applied in this section is based on the following assumptions. For the illumination conditions in the lithographic system, the effective illumination is assumed to consist of a circular centered monopole with a radius $\sigma$ that is very small compared to the numerical aperture (NA) of the lithographic projection lens and thus effectively generates a plane wave toward the reticle (Köhler illumination configuration). Upon transmission of the incident plane wave through a periodic structure on the reticle, a discrete number $w$ of diffracted beams are generated that lie within the entrance pupil NA of the projection lens (see also Fig. 1, where the special case of a PSG as object is illustrated). As a result, the aerial image of the diffraction grating can be considered as the superposition of a number $W$ of two-beam interferences, with $W=\left(\begin{array}{c}w \\ 2\end{array}\right)$. Each of these two-beam interferences gives rise to a cosine fringe in the aerial image, characterized by two parameters that are its phase offset and its amplitude. The two beams themselves are characterized by their complex-valued diffraction amplitudes given by $C_{1} \exp i \varphi_{1}$ and $C_{2} \exp i \varphi_{2}$, and by their reciprocal space coordinates denoted by $\left(h_{1}, g_{1}\right)$ and $\left(h_{2}, g_{2}\right)$, respectively. The interference of these two beams leads to a spatial frequency component of the aerial image, at the coordinates $\left(h=h_{1}-h_{2}, g=g_{1}-g_{2}\right)$, given by

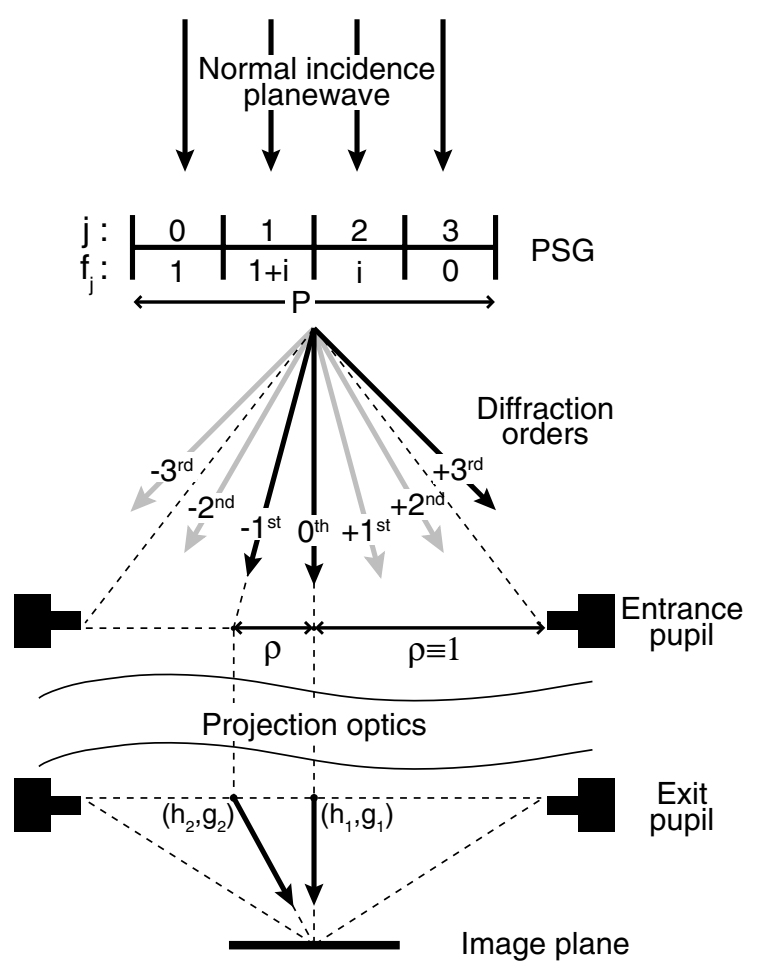

Fig. 1. PSG-based image formation. A schematic representation of image formation for a 1D PSG, having a unit cell with $N=4$ different taps and period $P$, is shown. The PSG at the reticle level is illuminated by a normal incidence plane wave. Each tap in the PSG unit cell has an effective scattering factor, $f_{j}$, with $j=0, \cdots, N-1$. The scattering factors shown correspond to the tap design values given in Eq. (11), where a complex valued $f_{j}$ indicates a relative phase shift for the light passing through that particular area of the unit cell. Interaction between the incident light and the PSG will generate a number of diffraction orders in the entrance pupil of the projection optics. Due to the complex scattering factors, destructive interference can take place and a number of diffraction orders are forbidden (shown in gray). We basically end up with only two non-zero orders within the NA, the zeroth and -1 st diffraction orders, and only these will propagate through the projection optics to contribute to the image. Note that the reciprocal space coordinates, $\left(h_{1,2}, g_{1,2}\right)$, as used in Eq. (1), are given by the coordinates of the two allowed diffraction orders in the pupil and that $\rho$ is the distance between the first diffraction order and the pupil center in normalized pupil coordinates.

$$
\begin{aligned}
\tilde{I}_{h, g}= & C_{1}^{2}+C_{2}^{2}+2 C_{1} C_{2} \cos \left[2 \pi\left(h_{1}-h_{2}\right) x\right. \\
& \left.+2 \pi\left(g_{1}-g_{2}\right) y+\varphi_{1}-\varphi_{2}+\Delta \Phi_{1,2}\right],
\end{aligned}
$$

where we will assume for now that $\varphi_{1}=\varphi_{2}$ (the $\varphi_{1} \neq$ $\varphi_{2}$ case is discussed at the end of Subsection 3.D). In this case, the overall phase offset of the cosine fringe, denoted by $\Delta \Phi_{1,2}$, is given by the difference of the wave-aberration function taken at the two spatial frequencies or diffraction angles in the exit pupil of the lens; the amplitude of the cosine fringe is determined by the diffraction amplitudes of the grating at mask level. The pattern shift of the aerial image is a complicated function of the phase shift and amplitudes for all the two-beam interferences that give rise to the aerial image. A directly interpretable 
and unambiguous relation between the pattern shift and the aberrations of the projection lens can be achieved when the aerial image is generated by only a single two-beam interference (see Figs. 1 and 2 ). In this case the observed pattern shift of the aerial image, $\Delta X_{\mathrm{AI}}$, is simply given by

$$
\Delta X_{\mathrm{AI}}=\frac{\Delta \Phi_{1,2} P}{2 \pi}
$$

with $P$ the period or pitch of the generated fringe pattern.

Given that, for the aimed aberration metrology, the entrance pupil consists of a single monopole centered at zero, a two-beam interference can only be realized by a diffraction grating at mask level that has at least one diffraction order that is "forbidden," i.e. has zero amplitude.

\section{B. Gratings with Forbidden Diffraction Orders}

The analysis of forbidden orders of a 1D diffraction grating with $N$ equidistant subdivisions or taps in its unit cell (see Fig. 1), and with for each $j$ th tap a respective scattering factor denoted by $f_{j}$, with $j=0,1, \ldots, N-1$, is typically carried out in terms of the unit cell's structure factor, which is, for the $k$ th diffraction order, given by, (e.g., Chapter 2, Eq. (46) in [3] , with $i^{2}=-1$ )

$$
F_{N}(k)=\sum_{j=0}^{N-1} f_{j} \exp \left\{2 \pi i \frac{j k}{N}\right\} .
$$

First, it should be noted that the tap-based scattering factors $f_{j}$ have to be complex valued in order to

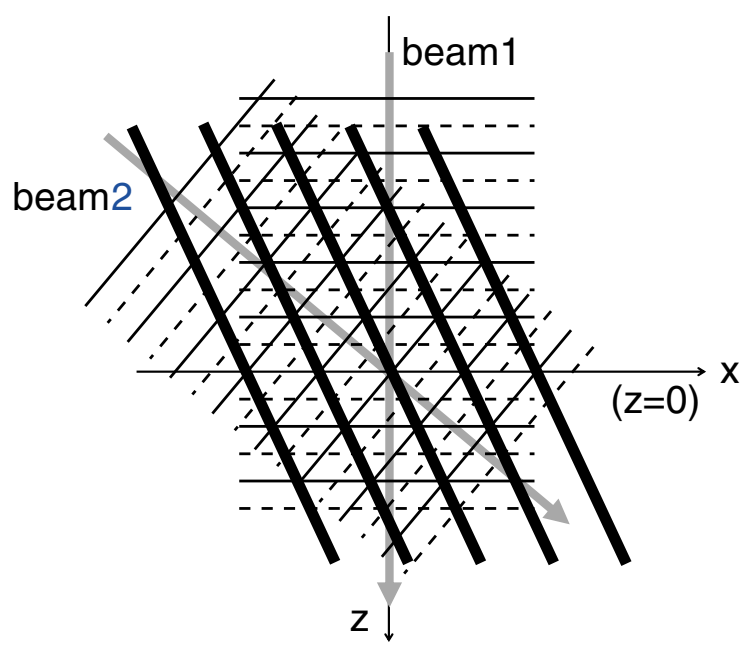

Fig. 2. Two-beam interference image formation. Beams 1 and 2 have equal wavelengths with dashed and solid lines representing tops and valleys of the plane wave, respectively. Where solid (or dashed) lines intersect, both beams are in phase and constructive interference takes place to form the image (interference fringes indicated by the thick black lines). A shift of the image plane in the $z$ direction (defocus) and/or a phase change of either beam will only shift the complete interference fringe in the image plane $(z=0)$ in the lateral $(x)$ direction. realize a forbidden order only at the $+k$ diffraction order position, while the $-k$ diffraction order has non-zero amplitude. Indeed, for real-valued scattering factors $f_{j}$, the symmetry relation $\left|F_{N}(-k)\right|=$ $\left|F_{N}(+k)\right|$ is always satisfied (also known as Friedel's law, a well-known property of the Fourier transform of a real-valued function). Next, the question of how many taps $N$ are to be distinguished within the unit cell should be addressed. For $N=2$, only the trivial solution for the set $f_{j}$ is obtained, equal to $\{1,1\}$, which actually implies a pitch halving. For $N=3$, only a real-valued solution for the set $f_{j}$ is obtained given by $\{1,1,1\}$, which does not qualify. For $N=4$, nontrivial complex-valued solutions are obtained for the set $f_{j}$. Note that without loss of generality, $f_{(j=0)}$ can be set equal to 1 . Possible sequences of diffraction orders in the reciprocal space unit cell $k=$ $0, \ldots, N$ are given in Table 1 . Cases 1 and 2 in Table 1 will be shown to be the (independent) relevant cases.

Case 1 requires that $\left|F_{(N=4)}(1)\right|=0$, which leads to the condition

$$
1+i f_{1}-f_{2}-i f_{3}=0 .
$$

This single equation on $\left\{f_{1}, f_{2}, f_{3}\right\}$ leaves $2 \mathrm{deg}$ of freedom. By making the choice $f_{2}=0=f_{3}$, the relation reduces to $1+$ if $f_{1}=0$, which yields the set $f_{j}$ given by $\{1, i, 0,0\}$, which is identified as Nomura's PSG [1].

Case 2 requires that both $\left|F_{(N=4)}(1)\right|=0$ and $\left|F_{(N=4)}(2)\right|=0$, which leads to the simultaneous conditions

$$
1+i f_{1}-f_{2}-i f_{3}=0
$$

$$
1-f_{1}+f_{2}-f_{3}=0 \text {. }
$$

The latter equations still leave 1 deg of freedom on $\left\{f_{1}, f_{2}, f_{3}\right\}$. By making the choice $f_{3}=0$, a non-trivial solution for the set $f_{j}$ is found to be $\{1,1+i, i, 0\}$. Clearly, by having two consecutive diffraction orders ( $k=1$ and $k=2$ ) that are forbidden, a larger range of pitches can be accommodated before a higher diffraction order enters the NA of the projection lens. In that sense, case 3 would hypothetically be even

Table 1. Seven Possible Sequences of Diffraction Orders for $N=4$, with "O" and " $X$ " Indicating Nonforbidden and Forbidden Diffraction Orders, Respectively, and the Top Row Giving the Indices $\boldsymbol{k}$ of the Diffraction Order

\begin{tabular}{llllll}
\hline$k$ & $0^{a}$ & 1 & 2 & 3 & 4 \\
\hline 1 & $\mathrm{O}$ & $\mathrm{X}$ & $\mathrm{O}$ & $\mathrm{O}$ & $\mathrm{O}$ \\
2 & $\mathrm{O}$ & $\mathrm{X}$ & $\mathrm{X}$ & $\mathrm{O}$ & $\mathrm{O}$ \\
3 & $\mathrm{O}$ & $\mathrm{X}$ & $\mathrm{X}$ & $\mathrm{X}$ & $\mathrm{O}$ \\
4 & $\mathrm{O}$ & $\mathrm{X}$ & $\mathrm{O}$ & $\mathrm{X}$ & $\mathrm{O}$ \\
5 & $\mathrm{O}$ & $\mathrm{O}$ & $\mathrm{O}$ & $\mathrm{X}$ & $\mathrm{O}$ \\
6 & $\mathrm{O}$ & $\mathrm{O}$ & $\mathrm{X}$ & $\mathrm{X}$ & $\mathrm{O}$ \\
7 & $\mathrm{O}$ & $\mathrm{O}$ & $\mathrm{X}$ & $\mathrm{O}$ & $\mathrm{O}$ \\
\hline
\end{tabular}

${ }^{a}$ The 0th order relates to the centered monopole illumination. 
more preferable, but its simultaneous conditions, given by

$$
\begin{aligned}
& 1+i f_{1}-f_{2}-i f_{3}=0, \\
& 1-f_{1}+f_{2}-f_{3}=0, \\
& 1-i f_{1}-f_{2}+i f_{3}=0,
\end{aligned}
$$

just allow for the trivial solution of the set $f_{j}$ given by $\{1,1,1,1\}$, which corresponds to an effective pitch that is a quarter of the original one. Similarly, case 4 corresponds to a pitch halving. Cases 5 and 6 are further equivalent to cases 1 and 2 , respectively, while case 7 obviously cannot lead to a situation with just two non-zero diffraction orders within the NA of the exit pupil. Concluding, for $N=4$, there are two solutions for the set $f_{j}$ that yield a diffraction pattern that can be used to generate a two-beam interference process within the NA of the optical system, given by

$$
\begin{aligned}
& \{1, i, 0,0\} \text { OXOOO, } \\
& \{1,1+i, i, 0\} \text { OXXOO. }
\end{aligned}
$$

Note that a complex scattering factor implies that light transmitted by the corresponding tap is phase shifted with respect to other taps. This is why a grating involving complex-valued taps is commonly referred to as a PSG and we will use this nomenclature to address this type of grating throughout the remainder of this paper.

\section{Sampling the Phase Aberration Function}

Using the grating designs given in Eqs. (10) and (11), it is possible to generate a diffraction pattern having only two non-zero (allowed) diffraction orders within the NA of the optical system, while all other diffraction orders are either zero (forbidden) or lie completely outside the NA. In this case, the image of the grating will be generated from a single two-beam interference and the simple relation given in Eq. (2) applies. Consequently, the phase difference of the aberration function evaluated at the position of the two allowed diffraction orders can be determined by measuring the displacement of the aerial image at the wafer level.

As the aberration function is probed at the location of the allowed diffraction orders, we can control the probing locations by varying the grating parameters. Assuming normally incident plane wave illumination, the 0th diffraction order of the grating will be generated at the center of the exit pupil, while the higher orders can be generated anywhere in the pupil by changing the pitch and rotation of the grating accordingly (see Fig. 3). Consequently, the gratings defined in Eqs. (10) and (11) can be used to sample the phase difference of the aberration function between the center position and another position

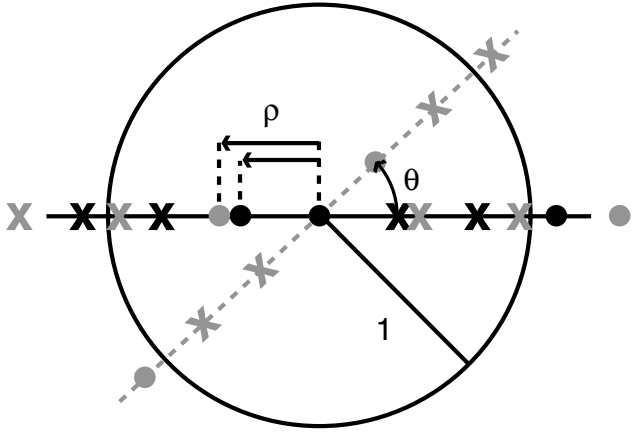

Fig. 3. PSG-based diffraction pattern control. Dots and crosses represent allowed and forbidden diffraction orders, respectively. Variation of the PSG pitch scales the diffraction pattern and changes the radial sampling position of the -1 st diffraction order. A rotation, $\theta$, of the PSG grating in the $x-y$ plane gives rise to an identical rotation of its diffraction pattern in pupil space.

elsewhere in the pupil, by controlling the 1st diffraction order position via the pitch and orientation of the grating.

However, the grating designs given in Eqs. (10) and (11) cannot be used to sample the entire pupil. This is because Eq. (2) is valid only in the case of pure twobeam imaging. This means that, for larger pitches, when the diffraction pattern is compressed relative to the size of the pupil, the condition should be satisfied that no non-zero higher diffraction orders enter the pupil. As a result, the following constraint applies on the maximum allowed grating pitch:

$$
P \leq \frac{k_{\min } \lambda}{\mathrm{NA}}
$$

where $k_{\min }$ specifies the lowest diffraction orders that should be kept outside the NA to satisfy the twobeam requirement. Note that $k_{\min }$ is different for the designs given in Eqs. (10) and (11); the first (Nomura) design has one allowed 2nd diffraction order implying $k_{\min }=2$, while the second design has both 2nd diffraction orders forbidden, thus allowing $k_{\min }=3$. As a result of the constraint given in Eq. (12), the aberration function cannot be sampled close to the center of the pupil (see also Fig. 5), with the excluded area being larger for the design in Eq. (10) than that in Eq. (11).

\section{Aberration Information from Overlay Measurements}

In the previous subsections it was explained that the image displacement of a PSG directly provides the phase difference between two discrete points of the aberration function. However, since the aberrations we intend to measure are expected to be very small, typically of the order of $5 \mathrm{~m} \lambda$ for well-corrected (lithographic) systems, it can prove quite challenging to measure the aberration-induced image displacement with a high enough accuracy to pick up these effects. Fortunately, the measurement of image displacements is a common task in the lithographic industry, known as overlay metrology, and we can exploit existing commercially available tools to 
measure our grating displacements accurately enough for the aimed aberration metrology described in this paper.

In the lithographic industry several different overlay metrology methods, which can be either diffraction-based or image-based, are available to determine the shift between layers, known as the overlay error. They all have in common that both layers for which the displacement should be compared should contain standard grating features from which it is possible to deduce the relative layer placement error. In the remainder of this paper we shall use an overlay measurement methodology known as diffraction-based overlay (DBO) to measure the PSG image displacements. We choose $\mathrm{DBO}$ as it is the method that currently provides the highest measurement accuracy and reproducibility based on a commercially available system (ASML YieldStar S/T-200). Nonetheless, the basic ideas and principles enabling the aberration metrology method presented in this paper can also be used to devise a similar aberration metrology method based on another overlay measurement technique.

In DBO, the displacement between two layers is assessed by including identical line-space (LS) gratings in each layer, which are then effectively printed on top of each other. What results is a stack with two gratings and possibly some other unstructured layers in between (see Fig. 4). The relative shift between the two gratings in the stack is determined via an asymmetry in the intensity of the +1 st and -1 st diffraction orders; the stack is illuminated by a spot after which the backreflected light, containing the first diffraction orders coming from the gratings, is collected on a CCD camera. Combining pupil images from two such grating stacks, both with a different yet known displacement between top and bottom gratings, it is then possible to accurately compute

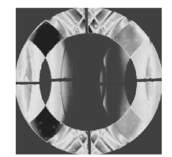

Shift in - direction

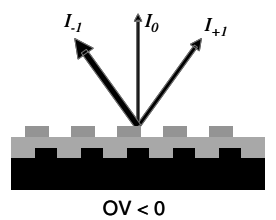

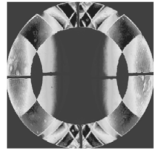

Symmetrical

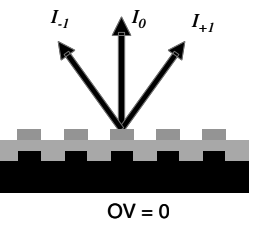

$\mathrm{OV}=0$

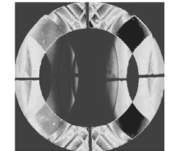

Shift in + direction

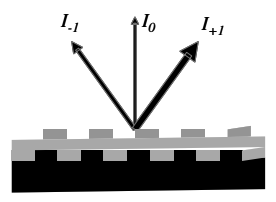

$\mathrm{OV}>0$
Fig. 4. Basic principle of DBO. A focused spot, with an annular pupil distribution, is used to illuminate a stack with two gratings. The backreflected light, containing diffraction orders, is collected and imaged on a CCD camera (top row images). In the case that both gratings are perfectly aligned, the image on the CCD will be symmetrical (center column), while if a displacement is present, the recorded CCD image is asymmetrical (left and right columns). Then using two such grating stacks, with known biases in overlay, the relative displacement between the top and bottom grating layers can be determined from the measured asymmetries in the free first diffraction orders reflected by both stacks. the overlay error between both layers (for DBO details see $[4,5]$ ).

The above-described standard DBO measurement can be adapted for our application as follows. We generate the top resist grating in the stacks from a PSG. As a bottom grating we use a standard LS pattern etched in silicon having the same pitch as the PSG and which we assume is manufactured and placed perfectly so that it may act as an absolute reference. In this case the overlay error measured between both gratings in the stacks, directly gives us the PSG image shift due to aberrations present during the exposure of the PSG layer (potential issues originating from a non-perfect bottom reference grating and/or additional overlay contributions coming from other sources than aberrations are discussed in Section 3 ).

A restrictive consequence of using DBO is that it imposes a constraint on the minimum pitch of the PSG gratings that can be used. If the pitch of the gratings in the stack is too small, their first diffraction orders will not lie within the NA of the wafer metrology sensor and, consequently, no overlay error can be determined. This leads to the following constraint on the minimum pitch:

$$
P>\frac{\lambda_{\mathrm{DBO}}}{D \mathrm{NA}_{\mathrm{DBO}}},
$$

where $\mathrm{NA}_{\mathrm{DBO}}$ and $\lambda_{\mathrm{DBO}}$ are the $\mathrm{NA}$ and radiation wavelength of the DBO tool and $D$ is a parameter controlling the amount of the first diffraction order present within the NA of the DBO tool. For $D \leq 2$, first diffraction order information is available; however, to allow accurate displacement measurements a typical value of $D=1.8$ is required. Consequently, the minimum grating pitch allowed by the DBO overlay measurement (with a YieldStar S/T-200; $\mathrm{NA}=0.95, \lambda=425 \mathrm{~nm})$ is $P \geq 249 \mathrm{~nm}$. In pupil space, this constraint translates to a sampling area in the pupil bounded by the radius $\rho \leq 0.57$, thus preventing us from sampling close to the edge of the pupil (see Fig. 므).

\section{E. Sampling Close to the Pupil Edge: 2D-PSGs}

The combination of the constraint on the minimum PSG pitch, which was given in Eq. (13), and the constraint on the maximum PSG pitch in Eq. (12) implies that the resulting pupil area that can be addressed (see Fig. 5) is clearly insufficient to collect enough phase information to successfully retrieve the aberration function. Also note that the pupil area that can be addressed by the design in Eq. (10) is completely contained by the area accessible with the design in Eq. (11), we will therefore exclusively use the latter design in the remainder of this paper and shall refer to it as 1D-PSG. To enlarge the fraction of the pupil that can be sampled using PSG targets in combination with $\mathrm{DBO}$, we shall now introduce a new type of two-dimensional PSGs (2D-PSGs) on top of the 1D-PSGs introduced in Subsection 2.B and will show how the additional design 


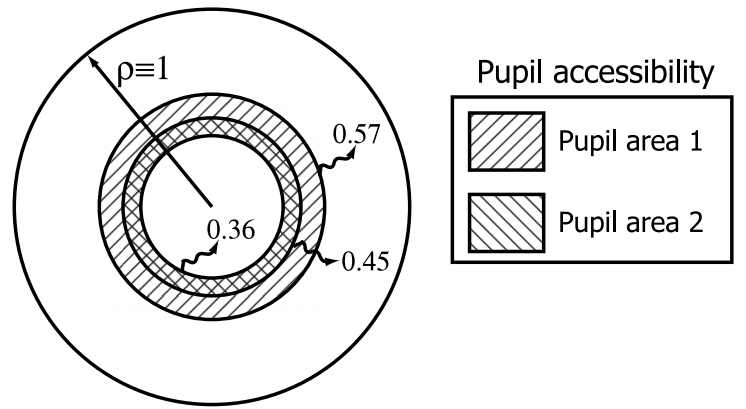

Fig. 5. Pupil accessibility of 1D-PSGs in combination with DBO metrology. The concentric circle at pupil radius 0.57 represents the maximum radial sampling position allowed by the constraint in Eq. (13) (for DBO with a YieldStar S/T-200; NA =0.95, $\lambda=4 \overline{25} \mathrm{~nm}$ ). The circles at pupil radii 0.45 and 0.36 represent the minimum radial pupil position allowed by the constraint in Eq. (12) for the PSG designs given in Eqs. (10) and (11), respectively. Consequently, phase information from the hatched Pupil area 1 can be obtained using the PSG design in Eq. (11) while the design in Eq. (10) can only be used to sample the smaller pupil subset labeled Pupil area 2.

freedom for these 2D-PSGs enables one to sample the aberration function near the pupil edge.

A 2D-PSG is defined in this context as having a typically oblique unit cell that allows for two (truly) diffracted beams within the NA of the projection lens, on top of the zeroth-order beam. This implies that there are three independent two-beam interferences that make up the aerial image at the wafer level; two of these three two-beam interferences comprise the zeroth-order beam, and the third two-beam interference occurs between the two truly diffracted beams [with non-zero reciprocal space coordinates $\left(h_{1}, k_{1}\right)$ and $\left(h_{2}, k_{2}\right)$, respectively]. The parameters of the $2 \mathrm{D}$ unit cell, i.e., lattice parameters $a$ and $b$ and the angle $\phi$ between them, are chosen such that the DBO metrology tool is only sensitive to the latter two-beam interference. This will be explained in more detail below.

The structure factor of the 2D unit cell, comprising $N$ taps per direction and $M=N^{2}$ objects, is given by

$$
F_{N}(h, k)=\sum_{j=0}^{M-1} f_{j} \exp \left\{2 \pi i\left(x_{j} h+y_{j} k\right)\right\}
$$

with $(h, k)$ the indices of the diffraction order, and $f_{j}$ and $0 \leq x_{j}, y_{j}<1$ the scattering factor and the (fractional) $2 \mathrm{D}$ coordinates of the $j$ th object, respectively. A 2D-PSG must then satisfy the following conditions (indicating a number of so-called forbidden diffraction orders):

$$
\begin{aligned}
F_{N}(-1,0) & =0, \\
F_{N}(0,1) & =0, \\
F_{N}(-1,-1) & =0, \\
F_{N}(1,1) & =0 .
\end{aligned}
$$

Table 2. Schematic Representation of the Diffraction Pattern Defined by the Conditions in Eq. (15), with "O" and " $X$ " Indicating Nonforbidden and Forbidden Orders, Respectively

\begin{tabular}{lccc}
\hline$(h, k)$ & -1 & 0 & 1 \\
\hline 1 & $\mathrm{X}$ & $\mathrm{X}$ & $\mathrm{O}$ \\
0 & $\mathrm{O}$ & $\mathrm{O}$ & $\mathrm{X}$ \\
-1 & $\mathrm{O}$ & $\mathrm{O}$ & $\mathrm{X}$ \\
\hline
\end{tabular}

These conditions yield a diffraction pattern with forbidden orders as shown in Table 2 .

Two structures that satisfy the conditions in Eq. (15) are shown in Fig. $\underline{6}$, both having four nontrivial (non-zero) scattering objects in the unit cell. Note that outside of the scattering object, the unit cell has no transmission. It should further be noted that some of the objects need to have a complexvalued scattering factor to enable the generation of forbidden diffraction orders, following a similar argumentation as in Subsection 2.B for the 1D-PSG case. The first type of unit cell has three different phases, $0,+90$ and $-90 \mathrm{deg}$, while the second type has only two different phases, 0 and -90 deg (parameters for both types are given in Table 3 ). The second type of 2D-PSG has the benefit that it uses only two phases, thus requiring fewer process steps in reticle manufacturing, and has better diffraction efficiency due to its larger scattering objects. In the remainder of this paper we shall exclusively use the second type and we shall simply refer to it as 2D-PSG.

Next, the 2D unit cell can be deformed from a square into an oblique one, thereby retaining the conditions of the forbidden orders. At this stage it proves more convenient to use an alternative, yet equivalent, representation of the 2D-PSG unit cell as given in the upper left of Fig. 7. The obliquity operation for the original unit cell in Fig. 6 is equivalent to a ratio change between the width, $\bar{A}$, and height, $B$, of the alternative design (see second row of Fig. 7). In addition to this, we can also apply an obliquity operation to the new unit cell definition, as shown
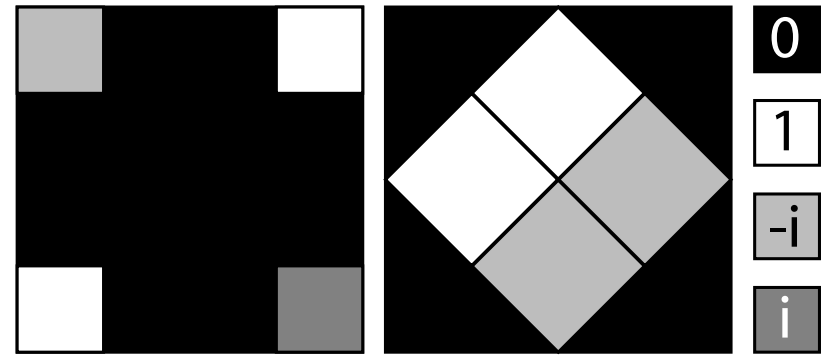

Fig. 6. Two 2D-PSG unit cell designs satisfying the conditions in Eq. (15). Both designs contain four scattering objects and their relative scattering factors are color coded according to the legend on the right; $i$ and $-i$ indicate relative phase offsets of +90 and $-90 \mathrm{deg}$, respectively. Note that the correct positioning and relative scattering factor of the objects in the unit cell are essential in achieving destructive interference for certain diffraction orders; the actual shape of the scattering objects is, in this respect, of minor importance. 
Table 3. Parameters Defining the Left and Right Unit Cells Shown in Fig. 6, Respectively, with the Four Scattering Objects Listed for Each Case

\begin{tabular}{cccc}
\hline$j$ & $f_{j}$ & $x_{j}$ & $y_{j}$ \\
\hline 0 & 1 & $\frac{1}{8}$ & $\frac{1}{8}$ \\
1 & $+i$ & $\frac{7}{8}$ & $\frac{1}{8}$ \\
2 & $-i$ & $\frac{1}{8}$ & $\frac{7}{8}$ \\
3 & 1 & $\frac{7}{8}$ & $\frac{7}{8}$ \\
\hline$j$ & $f_{j}$ & $x_{j}$ & $y_{j}$ \\
0 & $-i$ & $\frac{1}{2}$ & $\frac{1}{4}$ \\
1 & $-i$ & $\frac{3}{4}$ & $\frac{1}{2}$ \\
2 & 1 & $\frac{1}{4}$ & $\frac{1}{2}$ \\
3 & 1 & $\frac{1}{2}$ & $\frac{3}{4}$ \\
\hline
\end{tabular}

in the bottom left of Fig. 7. The impact of these transformations on the gratings diffraction pattern is shown on the right of Fig. 7. Going from the first to the second row, it can be observed that a ratio change between $A$ and $B$ compresses the diffraction pattern in one direction, effectively bringing the allowed diffraction orders $(-1,0)$ and $(0,1)$ closer together. On the other hand, the obliquity transformation causes the two allowed orders to be generated at different radial distances from the pupil center, and this will prove to be an essential feature in measuring spherical aberration components later on. Full details on the relation between the 2D-PSG unit cell parameters and the resulting position of its allowed diffraction orders are provided in Appendix B.

Since the diffraction pattern of a 2D-PSG contains three allowed orders within the pupil, its aerial image is comprised of a superposition of three two-beam interference fringes [with their respective reciprocal space coordinates $(h, k)]$ :

$$
\begin{aligned}
(0,0)-(1,0) & =(-1,0) \\
(0,0)-(0,-1) & =(0,1) \\
(1,0)-(0,-1) & =(1,1)
\end{aligned}
$$

Now the important observation to be made here is that the first two fringes in Eq. (16), involving the zeroth order, will have a small period, as the distance between the orders is relatively large, and will therefore not satisfy the constraint in Eq. (13). On the other hand, the third fringe in Eq. (16) stems from interference between orders $(0,-1)$ and $(1,0)$ and, since these orders are relatively close together, generates a fringe $\left(h=h_{1}-h_{2}, k=k_{1}-k_{2}\right)$ with a large enough period to be observed by the DBO tool. As a result, the complicated $2 \mathrm{D}$ resist grating generated from a 2D-PSG will be observed by the DBO tool as a simple $1 \mathrm{D}$ pattern as if it was generated exclusively from interference between orders $(0,-1)$ and $(1,0)$. Moreover, it turns out that even the simple relation given in Eq. (2) remains valid for the fringe generated by the orders $(0,-1)$ and $(1,0)$. Then, by applying a resist grating imaged from the 2D-PSG as the top grating in an overlay stack, and using a

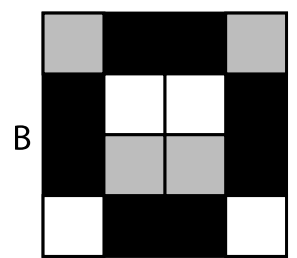

A

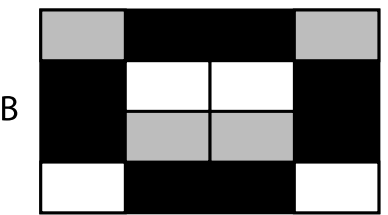

A

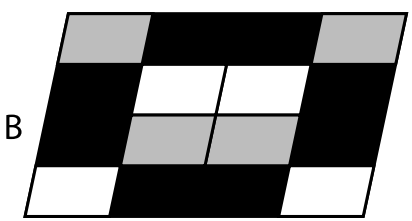

A
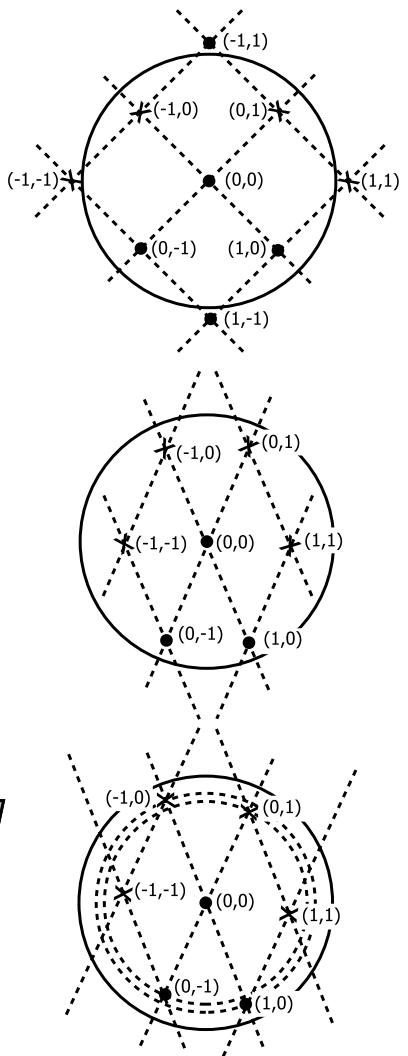

Fig. 7. 2D-PSG unit cells and their corresponding diffraction patterns (dots and crosses represent allowed and forbidden orders, respectively). The first row pertains to the nominal 2D-PSG design, which is equivalent to the minimum unit cell given on the righthand side of Fig 6 . In the second row, the unit cell is transformed such that $A \neq B$, resulting in a compression of the diffraction pattern in one direction. In the third row, the obliquity transformation is applied, resulting in the generation of the orders $(1,0)$ and $(0,-1)$ at different distances from the pupil center. Note that the diffraction orders $(1,0)$ and $(0,-1)$ are the two truly diffracted beams. The orders that are forbidden because of the 2D-PSG are $(-1,0),(0,1),(-1,-1)$, and $(1,1)$. Some higher orders, for example $(-2,-2)$ and $(2,2)$, are not forbidden, but remain outside of the NA of the optical system. Note that the nomenclature used here, and throughout this paper, to indicate a diffraction order pertains to the minimum unit cell as defined in the right-hand side of Fig. $\underline{6}$.

LS grating etched in silicon matched to the DBO observable 1D component, the phase difference between two points close to the pupil edge can again be measured as an overlay value.

Similar as in the $1 \mathrm{D}$ case, a multitude of $2 \mathrm{D}-\mathrm{PSG}$ gratings at different orientations at mask level can be used so that the aberration function is sampled at different azimuths in the pupil. Moreover, some freedom in the allowed unit cell parameters can be used to achieve additional measurement diversity (for more details, see Appendix B). Consequently, combining 1D-PSGs and 2D-PSGs, it is possible to collect differential phase information on the aberration function for the larger part of the pupil via DBO measurements (see Fig. 8). This, in principle, opens up the route toward a method capable of estimating the aberrational state of a lithographic system based on a large number of overlay measurements. 


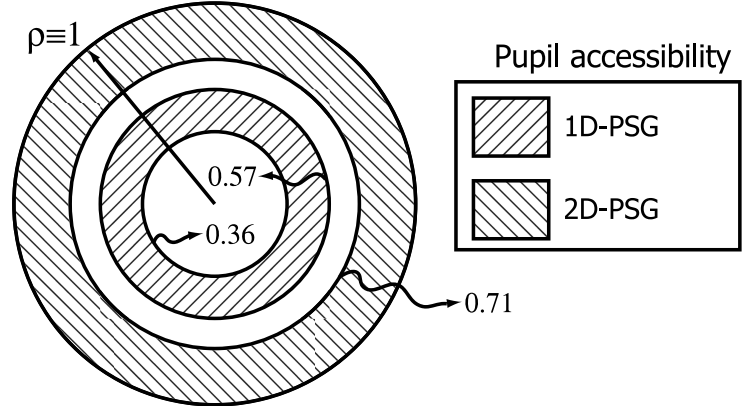

Fig. 8. Pupil accessibility of 1D- and 2D-PSGs in combination with DBO.

\section{F. PSG-OVL Aberration Model}

In the previous subsections it was explained that the phase difference, measured as an overlay value, between different points in the pupil can be obtained by varying the type, unit cell parameters, and orientation of the PSG targets used in the overlay stack. In this way, by using both $1 \mathrm{D}$ - and 2D-PSGs with varied parameters, it is in principle possible to collect enough information on the aberration function to retrieve it. However, to estimate the aberration function based on differential phase information we need to define an appropriate model and aberration representation and for this purpose we proceed as follows.

Let $\Phi(\rho, \theta)$ be the aberration function defined on the pupil in normalized polar coordinates $(0 \leq \rho \leq 1$ and $0 \leq \theta \leq 2 \pi)$. The classical Zernike expansion of the aberration function is then given by

$$
\Phi(\rho, \theta)=\sum_{n, m} \alpha_{n}^{m} Z_{n}^{m}(\rho, \theta)
$$

where $\alpha_{n}^{m}$ denotes a Zernike coefficient for integer $n$, $m$ such that $n-|m|$ is even and non-negative and $Z_{n}^{m}(\rho, \theta)$ denotes a classical Zernike circle polynomial defined as

$$
Z_{n}^{m}(\rho, \theta)=R_{n}^{|m|}(\rho) \begin{cases}\cos (|m| \theta) & \text { for } m \geq 0 \\ \sin (|m| \theta) & \text { for } m<0\end{cases}
$$

Note that we choose to use the double index Zernike convention here as it will prove mathematically more convenient to construct an appropriate model. On the other hand, we will use the single index Zernike convention in the text and figures to refer to specific aberration terms. A conversion rule, to go from the single index Zernikes (FRINGE convention) to the double index, and vice versa, is provided in Appendix A.

Next, let $\left(\rho_{1}, \theta_{1}\right)$ and $\left(\rho_{2}, \theta_{2}\right)$ be the polar pupil coordinates of the two nonforbidden diffraction orders of a given PSG and let $\Delta \Phi_{1,2}$ be the phase difference of the aberration function evaluated at these positions:

$$
\Delta \Phi_{1,2}=\Phi\left(\rho_{2}, \theta_{2}\right)-\Phi\left(\rho_{1}, \theta_{1}\right)
$$

Then, using Eqs. (2), (17), and (19), we can write the aberration induced overlay error, OVL, for the current PSG as

$$
\begin{aligned}
\mathrm{OVL} & =\Delta X_{\mathrm{AI}}=\frac{\Delta \Phi_{1,2} P}{2 \pi} \\
& =\frac{P}{2 \pi} \sum_{n, m} \alpha_{n}^{m}\left[Z_{n}^{m}\left(\rho_{2}, \theta_{2}\right)-Z_{n}^{m}\left(\rho_{1}, \theta_{1}\right)\right],
\end{aligned}
$$

with the Zernike coefficients $\alpha_{n}^{m}$ describing the aberration state of the imaging system.

If the aberration state of a system is unknown, the expression in Eq. (20) allows one to devise a linear system of equations relating the unknown Zernike coefficients, $\alpha_{n}^{m}$, to measured overlay values. Estimates for the $\alpha_{n}^{m}$ are then obtained by solving the standard least-square minimization problem defined by $[6]$ :

$$
\alpha_{\min }=\underset{\alpha}{\arg \min }\left\|E \alpha-\mathrm{OVL}_{\text {meas }}\right\|^{2},
$$

with $\alpha \in \mathbb{R}^{G}$ the vector of unknown Zernike coefficients, $\mathrm{OVL}_{\text {meas }} \in \mathbb{R}^{H}$ the vector of measured overlay values per PSG target, and $E \in \mathbb{R}^{H \times G}$ a $2 \mathrm{D}$ matrix containing the model predicted overlay per Zernike term per PSG as constructed using Eq. (20). Note that $H>G$.

\section{G. Proof-of-Principle in Simulation}

In Subsection 2.F it was shown how to relate the measured PSG overlay values to the aberrations of the lithographic system. Based on this model, a linear system of equations can be constructed that, upon solving, provides estimates for the Zernike coefficients representing the aberration function. To evaluate the potential of our method we have executed the following numerical experiment.

1. A set of 420 PSG targets (with a set of matching 1D LS reference gratings at the bottom of the grating stack) is defined, aimed at sampling throughout the pupil while satisfying the constraints given in Eqs. (12) and (13) and Appendix B.

2. Using a commercial lithographic simulator [Prolith (64-bit) Version 14.2.0.29], the aerial image of all PSG targets is simulated under realistic exposure conditions, given an arbitrary aberration state of the lithographic system.

3 . The images of the reference gratings are simulated in the same way. However, since these gratings are on a different layer, the aberration conditions during their exposure are possibly different, which is accounted for in the simulation by using another (unknown) aberration state.

4. The aberration-induced overlay error per PSG target is then obtained as the shift between the aberrated PSG and the reference grating aerial image.

5. Finally, the simulated overlay values serve as input to the least-square minimization problem given in Eq. (21), which, upon solving, provides 

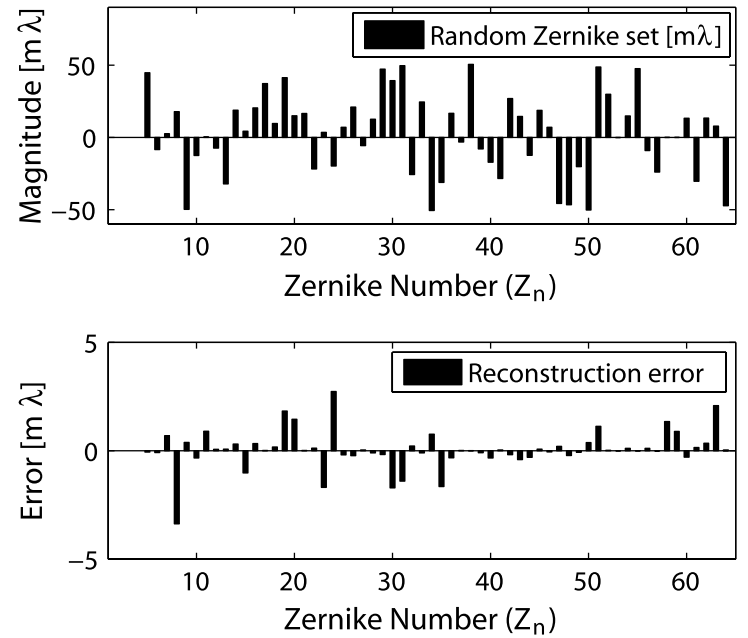

Fig. 9. Numerical proof-of-principle. A set of 60 Zernikes $\left(Z_{5}\right.$ to $\left.Z_{64}\right)$ is defined with random values between -50 and $+50 \mathrm{~m} \lambda$ (top row). For this Zernike set, the aberration- induced shift of all 420 PSG targets is computed and the resulting simulated values serve as input to the minimization problem defined in Eq. (21). The bottom row presents the error in the estimated Zernike coefficients, which is better than $5 \mathrm{~m} \lambda$.

estimates for the $\alpha_{n}^{m}$ describing the aberration state as defined during the simulation of the PSG images.

The results of the above-described numerical experiment are presented in Fig. 9. In the top figure, the arbitrary set of 64 Zernikes used for this simulation is shown. In the bottom panel, the error per estimated Zernike is displayed. These simulation results clearly show that the Zernike coefficients describing the aberrational state of a lithographic system can in principle be estimated based on measured relative image displacements. Note that an error is observed in the estimated coefficients due to the fact that the various constraints do not allow the pupil to be sampled optimally and because the simulation involved a reference grating that was produced under different aberration conditions (random set with the same order of magnitude as shown in the top of Fig. 9). Nevertheless, the presented simulation shows that the method set out in this paper is capable of estimating the aberrational state of a lithographic system under these conditions, with an accuracy better than $5 \mathrm{~m} \lambda$.

\section{Experimental Challenges and Refinements}

In the previous section, the basic concepts and a numerical proof-of-principle were presented for our wafer-based aberration metrology method. In the present section, we discuss the complications that arise when the proposed method is implemented experimentally, and we will evaluate the impact of these potential issues on the overall performance of the method. Where necessary, refinements of the PSG-OVL model and measurement procedure are proposed in order to minimize their negative impact.

\section{A. Finite Illumination Source}

During the discussion of the basic measurement principles in Section 2 it was assumed that the PSG targets at the reticle are illuminated coherently by a plane wave at normal incidence. However, in reality all lithographic systems have a finite source, commonly combined with a Köhler illumination system. In such an illumination configuration, every source point generates a plane wave at a slightly different angle toward the reticle and, consequently, the reticle image can be considered as the sum of many coherent contributions. In addition to this, the source is imaged in the pupil planes of the projection optics. This implies that light incident on a PSG, originating from different points on the source, will generate diffraction orders at slightly different spatial frequencies in the pupil and consequently will accumulate a different relative phase on propagation through the projection optics if aberrations are present (see also Fig. 10). Under these conditions, Eq. (2) is still valid for predicting the aberration-induced fringe displacement for a single coherent contribution, but does not give a good prediction for the overall displacement of the total PSG image, which consists of the superposition of all differently displaced coherent contributions. Consequently, the method presented in Section 2 will also not provide optimal aberration estimates for realistic lithographic systems due to the impact of their finite sources.

A straightforward way to reduce the impact of the finite source is to make it smaller so that it better approximates the coherent (single point) illumination case. In modern day lithographic systems, this is possible via a user-defined effective source in the entrance pupil; for example a small centered disk. However, the minimum area of such an effective

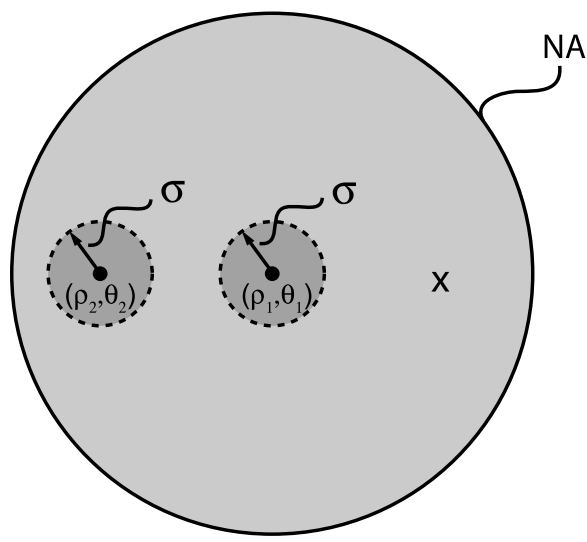

Fig. 10. Schematic representation of the projection optics exit pupil for the case of a 1D-PSG in combination with an on-axis finite monopole effective source. The monopole source, the size of which is defined by the radius $\sigma$ relative to the NA of the projection system, is convoluted with the PSG diffraction orders at locations $\left(\rho_{1}, \theta_{1}\right)$ and $\left(\rho_{2}, \theta_{2}\right)$. As the source is assumed spatially incoherent, each point on the resulting diffraction order disks can interfere only with its corresponding point on the other disk and each of these point pairs effectively generates a single coherent contribution. 
source is constrained, because making it too small either increases the exposure time too much or requires the energy density to be so high that the optics can be damaged. Consequently, the source representation in the pupil cannot be small enough to allow for an accurate coherent treatment of the problem and we need to accommodate for the finiteness of the effective source through the PSG-OVL model, for which we proceed as follows.

We assume the effective source in the pupil to be a small disk (monopole), with radius $\sigma$, that is spatially incoherent and uniform. In addition, it is assumed that the diffraction efficiency of the scattering objects at the reticle do not vary with the angle of incidence of the light. The total PSG image is then obtained as the incoherent summation of all fringe intensities while integrating over the source, and the effective aberration-induced shift simply becomes the average of the individual fringe displacements. In this case, an expression similar to Eq. (2) can be obtained, relating the overall PSG-image shift, in the case of a finite source, to the average phase difference between two areas in the pupil:

$$
\Delta X_{\mathrm{AI}}=\frac{P}{2 \pi}\left(\bar{\Phi}_{\sigma}\left(\rho_{2}, \theta_{2}\right)-\bar{\Phi}_{\sigma}\left(\rho_{1}, \theta_{1}\right)\right),
$$

where $\bar{\Phi}_{\sigma}$ denotes the average of the aberration function, taken over a disk with radius $\sigma$ at the diffraction order positions $(\rho, \theta)$ (see Fig. 10). Next, the phase average over a disk-shaped area in the pupil can also be written in terms of Zernikes and we can finally write an equivalent expression for Eq. (20) in which the finiteness of the source is accounted for:

$$
\begin{aligned}
\mathrm{OVL} & =\Delta X_{\mathrm{AI}}=\frac{P}{2 \pi}\left(\bar{\Phi}_{\sigma}\left(\rho_{2}, \theta_{2}\right)-\bar{\Phi}_{\sigma}\left(\rho_{1}, \theta_{1}\right)\right) \\
& =\frac{P}{2 \pi} \sum_{n, m} \alpha_{n}^{m}\left[\bar{Z}_{n ; \sigma}^{m}\left(\rho_{2}, \theta_{2}\right)-\bar{Z}_{n ; \sigma}^{m}\left(\rho_{1}, \theta_{1}\right)\right],
\end{aligned}
$$

with $\bar{Z}_{n ; \sigma}^{m}(\rho, \theta)$ the average of a Zernike function taken over a possibly truncated disk with radius $\sigma$ at the pupil position $(\rho, \theta)$. Accurate computation of Zernike function averages required in Eq. (23) is discussed in Appendix C. It should be noted that the 2D-PSGs introduced in Subsection 2.E require some additional attention. As they are meant to sample the aberration function near the pupil edge, we also have to deal with the case that, for either one or both of the relevant interfering diffraction orders, the averaging disk is partly outside the NA of the lithographic system. Since the effective source is considered incoherent, a point on the averaging disk will contribute only if the corresponding points in the other diffraction order also lie within the NA of the system. Source points for which only one diffraction order lies within the NA cannot interfere and, consequently, produce only a spatially uniform yet irrelevant contribution to the image. In Fig. 11 the common area of both allowed diffraction orders is

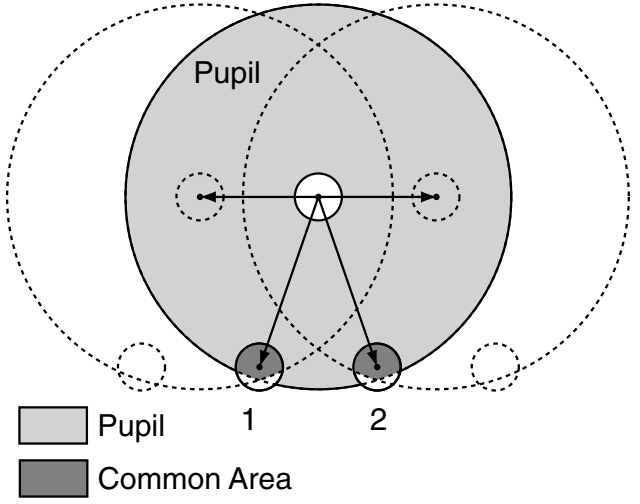

Fig. 11. 2D-PSG diffraction orders partly outside the pupil. A point on one diffraction order disk can only interfere, and thus contribute to the overall image, if its corresponding point on the other disk also lies within the pupil. As a result, the effective diffraction order phase is obtained by integration over the common area of both orders, which will be an irregular shaped area when one, or both, diffraction orders lie partly outside the pupil. Systematically computing the average phase for such areas is dealt with in Appendix C.

indicated for a general case that both orders are cut differently by the NA limited pupil. The task of computing the relative phase for the resulting strangely shaped common area, which is indicated in Fig. 11, is also dealt with in Appendix C.

In Fig. 12 the impact of a finite source (on-axis monopole) on the effective phase of a diffraction order area is shown. On the left, the effective diffraction order phase, for Zernike function $Z_{36}$, is shown as a function of the source radius, $\sigma$, for four radial positions of the averaging disk in the pupil. It can be seen that the effective diffraction order phase deviates substantially from the coherent case $(\sigma=0)$, already for modest illumination $\sigma$, clearly showing the necessity of the finite source correction. The righthand figure compares the effective diffraction order phase for $\sigma=0.0$ (coherent case) and $\sigma=0.122$
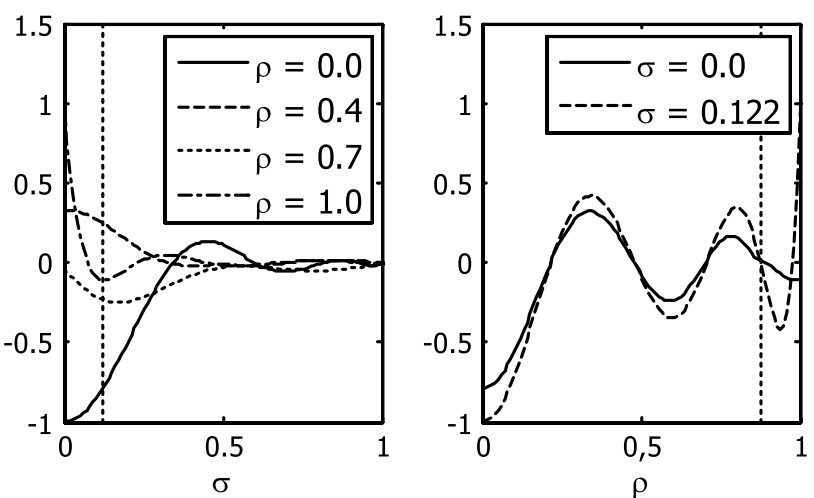

Fig. 12. Finite source impact. Assume a phase distribution in the pupil, defined by Zernike function $Z_{36}$. On the left, we plot $Z_{36}$ averaged over a disk as a function of the averaging disk radius, $\sigma$, for four different radial positions in the pupil. On the right, the disk average is plotted as a function of its radial position to illustrate the impact of a finite monopole source $(\sigma=0.122)$ compared to the coherent case $(\sigma=0.0)$. 
(realistic lower bound for the $\sigma$ in a lithographic system). Especially for averaging areas near the pupil edge $(\rho \rightarrow 1)$, as encountered for the 2D-PSGs, the difference between the coherent and finite source models is non-negligible.

\section{B. Asymmetric Resist Gratings}

Another experimental complication comes from the fact that the PSGs are imaged by a two-beam process into a layer of resist with a finite thickness. As a result of this, the resist grating formed from a 1D-PSG will be asymmetric (see left-hand side of Fig. 13). In the 2D-PSG case, where the image is formed by mutual interference among three orders, the resulting resist grating looks even more exotic, with effective grating lines built up from tilted pillars (see righthand side of Fig. 13). These irregular grating shapes introduce an offset in the asymmetry signal of a DBO measurement and, consequently, also give rise to a process and PSG dependent, yet constant, offset in the measured overlay values. When not accounted for, these offsets introduce very large errors on the reconstructed spherical aberration terms (see also Fig. 16). Fortunately, these artifacts are independent of the aberrations that we want to determine, thus making it possible to remove them via a calibration step. For this purpose one can execute the presented aberration metrology method on a very wellcorrected lithographic system and assume that the overlay values measured on that system for all PSGs are exclusively due to the PSG grating asymmetries. Now by subtracting these measured overlay residuals, as a reference measurement, from any subsequent PSG-OVL measurement, one effectively removes the Zernike reconstruction error coming from the resist grating asymmetry. The price that is paid for this is that the reconstructed Zernikes are now no longer independent and describe the aberration state of the unknown system relative to the reference system.
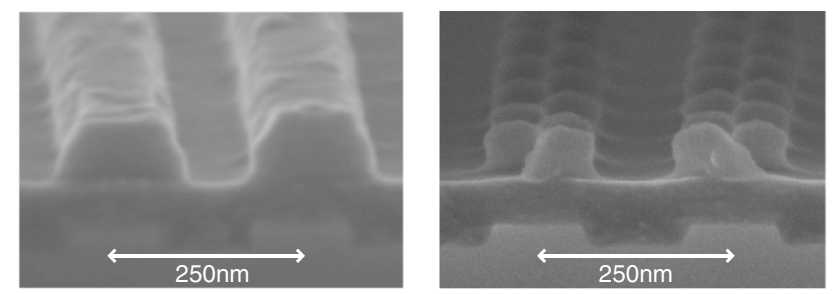

Fig. 13. Cross-section scanning electron microscopy (SEM) images. On the left a cross-section SEM image of a 1D-PSG wafer stack is shown and one can observe a significant difference in side-wall angle between the sides of the grating lines. On the right, a cross-section SEM image of a 2D-PSG wafer stack is shown. It is clearly observed that, although the line appears to be built up from tilted pillars, the 2D-PSG generates effectively a $1 \mathrm{D}$ grating with a pitch matched to the bottom grating. The fine structure in the grating line is subresolution for the DBO measurement, but can possibly contribute to the asymmetry signal generated by the whole stack.

\section{Other Overlay Contributions}

In this paper, the aberrations are estimated based on measured overlay values. Therefore, it can be easily understood that overlay contributions coming from sources other than aberrations directly propagate into the Zernike reconstruction error. Possible other sources contributing to the overlay are:

\section{Mechanical overlay.}

The actual displacement between different layers of the wafer, caused by wafer stage and reticle stage positioning errors.

\section{Reticle registration errors.}

The misplacement of features on the reticles with respect to the reticle designs.

\section{Reticle and wafer deformations.}

Temperature gradients, (thermo-)mechanical strain and/or processing can cause the reticle and wafer to deform, introducing position-dependent relative displacements of the features they contain.

In its simplest form, mechanical overlay imposes a relative $(x, y)$ position shift between features in two different layers of the wafer; this shift is identical throughout our small-sized target area in the die. In our method, where we measure the grating displacement in the direction of the grating vector only, the mechanical overlay contribution is given by the projection of the vector $(x, y)$ on the grating vector. As a result, mechanical overlay gives a pitchindependent contribution to the PSG grating displacement, but does vary with the PSG orientation. It turns out that the mechanical overlay fingerprint, the fingerprint being the combined response of all PSG targets to a given overlay source, is identical to that of a tilt aberration (linear combination of $Z_{2}$ and $Z_{3}$ ), and we may conclude that mechanical overlay does not contribute to the relevant Zernikes $\left(Z_{t}\right.$ for $t>3$ ).

The second overlay contribution listed is coming from the reticle. If features on the reticle are not placed exactly at the position defined in the reticle design, this displacement contributes directly to the observed overlay for the displaced target. Since the overlay is defined as the displacement between two gratings in two different layers imaged from two different reticles, the combined overlay contribution of both reticles is in fact the target specific relative displacement between its bottom and top gratings, also known as the registration error between the two reticles. This registration error, which is basically a manufacturing defect that remains static during the lifetime of a reticle, is commonly measured during manufacturing of a reticle set and could therefore be compensated for in the PSG-OVL model. However, this is not even necessary when a calibration as described in Subsection 3.B is performed, as this calibration step also automatically compensates for the static overlay contribution induced by the reticle registration error. Other reticle defects also contribute to the Zernike reconstruction 
error of our method, but these are discussed in more detail in the next subsection.

The final contribution listed above is trickier. The mentioned deformations introduce overlay errors according to the same mechanism as the reticle registration error, but in this case depend on environmental and exposure conditions, implying that they are not static and are, therefore, not automatically removed by the calibration. The overlay contribution generated by wafer and reticle deformations is position dependent and can in principle result in an arbitrary overlay fingerprint for a PSG-target set. However, temperature, strain, and processing deformations typically give rise to overlay contributions that vary slowly over the wafer. By placing all PSG targets required for our method close together, we can minimize the variation and, therefore, the impact of these deformations to an acceptably low level. If this is insufficient, one may resort to complex computational models to predict temperature-induced deformations, but this has not been investigated further in this paper.

\section{Reticle 3D Effects and Manufacturing Errors}

A typical lithographic reticle consists of a quartz substrate on which a thin layer of chromium is deposited to make it opaque. By selectively removing chromium using electron beam lithography, a desired binary pattern can be transferred. In addition to this, our PSG designs require that certain transmissive areas on the reticle are assigned a different phase, and this can be achieved by selectively etching those areas into the substrate. Combined, this allows us to manufacture a reticle with PSG targets according to the designs described in Section 2. However, since the resulting PSG feature sizes on the reticle are of the same order of magnitude as the illumination wavelength and the grating cannot be considered optically thin, the diffraction efficiency of the PSG targets is not predicted well by the Kirchhoff diffraction model. Instead, a rigorous 3D-diffraction model should be applied to correctly account for the lightmatter interactions taking place at the reticle. In the first two rows of Fig. 14, the simulated diffraction pattern is shown for these two cases, clearly illustrating that the forbidden diffraction orders of the nominal PSG design are no longer zero when the 3D structure of the reticle is accounted for. At the bottom row of Fig. 14, however, it is shown that this issue can be solved by optimizing the nominal PSG design so that it compensates for the 3D-diffraction effects to again achieve forbidden diffraction orders.

In addition to the 3D-diffraction effects discussed above, reticle manufacturing errors can also contribute to unwanted energy in the forbidden orders. These are, for example, corner rounding, under-etch, and nonvertical etch side walls, and they can all modify the diffraction efficiency of the features on the reticle (see also Fig. 15). As these imperfections are difficult to predict and to parameterize, it is impractical to accurately estimate their impact via

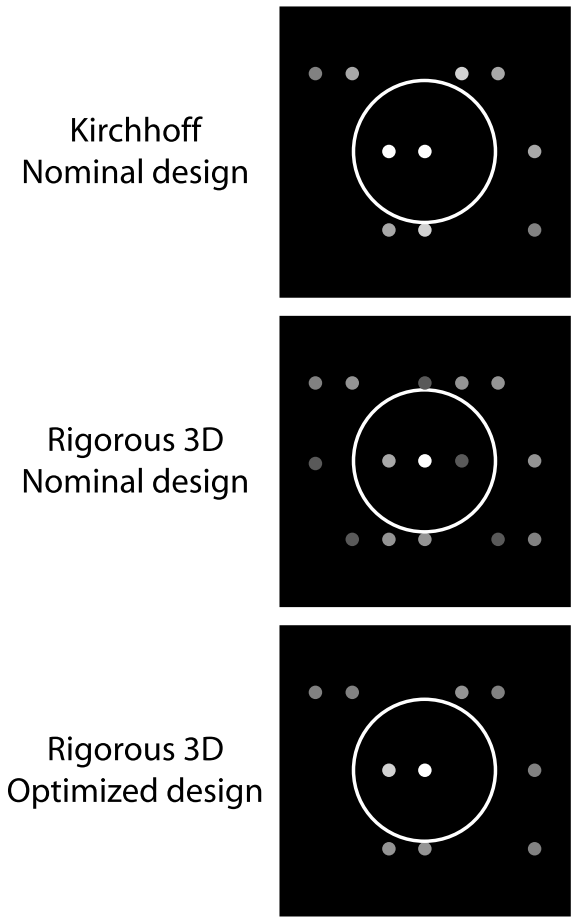

Fig. 14. Computed diffraction patterns for the nominal 1D-PSG defined in Eq. (11). The top image shows the diffraction pattern computed according to Kirchhoff, with, as expected, only two non-zero orders within the NA (indicated by the white circle). The middle image shows the diffraction pattern for the same target when correctly accounting for the reticle $3 \mathrm{D}$ effects. In this case, three non-zero orders are predicted within the NA, destroying the pure two-beam process. In the bottom image, the rigorously computed diffraction pattern is shown for an optimized 1D-PSG design, showing that the pure two-beam process is restored.

computational means. Instead, one may choose to further optimize the PSG designs for these effects experimentally. This would be done by making a test reticle containing PSGs for different combinations of design and processing parameters. Next, the amount of energy in the forbidden orders is assessed by measuring the diffraction pattern, which can, for example, be done using a Zeiss AIMS system. The combination of parameters for which the forbidden order strength is minimal is then used to manufacture the actual measurement reticle.

In reality, the forbidden diffraction orders will never be completely zero. The first order effect of a small to moderate non-zero forbidden order is that it reduces the sensitivity of our proposed method for aberrations having an even azimuthal dependence ( $\alpha_{n}^{m}$ with $m$ is even), while not influencing the sensitivity of odd aberrations ( $m$ is odd). However, since we can, in principle, measure the relative forbidden-order strengths of all PSGs with an AIMS microscope, this effect can also be accounted for in the model by adjusting the sensitivity matrix $[E$ in Eq. (21)] accordingly. Nevertheless, it remains best practice to strive for the lowest possible amount of energy in the forbidden orders to avoid higher order effects and to have the highest possible sensitivity for all aberrations. 

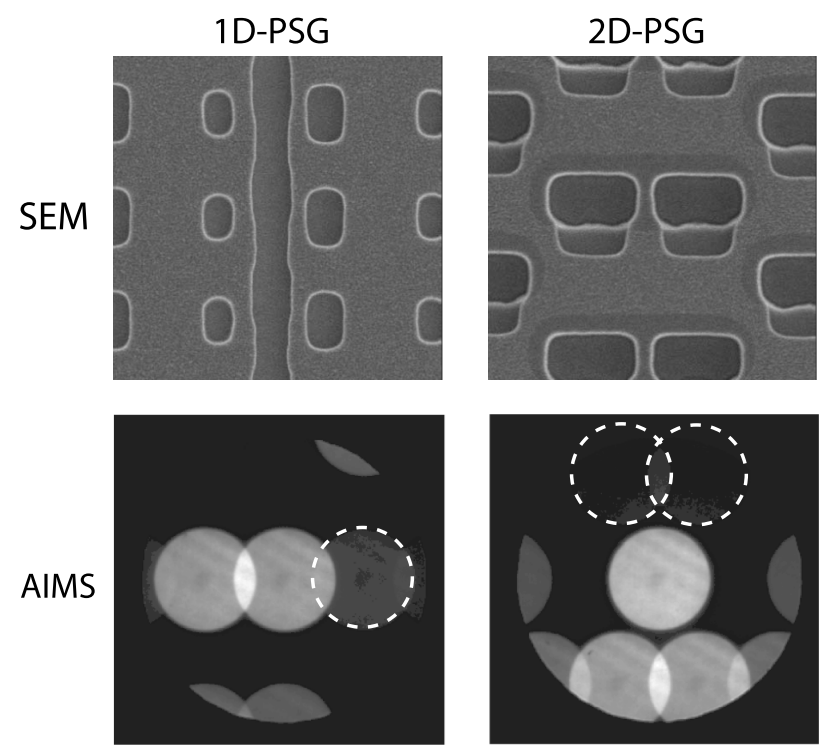

Fig. 15. Illustration and impact of reticle manufacturing defects. The top row shows SEM images of typical 1D- and 2D-PSGs on the reticle. The 1D SEM image was taken before the phase etch step, while the 2D SEM image was taken afterward. One can observe significant corner rounding and additional deformations due to the phase etch into the substrate. In the bottom row, typical diffraction patterns for 1D- and 2D-PSGs are shown as measured by an AIMS. Due to the writing and etching defects, the intended forbidden orders (indicated by dashed circles) are non-zero. Note that the monopole source of the AIMS is relatively large, resulting in overlapping orders and higher diffraction orders showing up at the pupil edge that are of no concern in the actual experiment in the lithographic system where a much smaller source is used.

Even in the case of perfectly forbidden orders, there is one other reticle characteristic that can impact the overlay measured in our method and has not yet been discussed. This is the relative phase of the allowed diffraction orders, $\varphi_{1}$ and $\varphi_{2}$, as occurring in Eq. (1). Looking at Eq. (1), it can be understood that if $\varphi_{1}-\bar{\varphi}_{2} \neq 0$ this directly adds up to, and cannot be distinguished from $\Delta \Phi_{1,2}$, the aberration-induced phase contribution and, consequently, contributes directly to the error of the Zernikes reconstructed by our method. Furthermore, the diffraction order phase is not easily measured using existing semiconductor equipment, which implies that a model-based correction of this artifact is not feasible. But again, this reticle effect can also be assumed static, assuring that it is also effectively removed by the calibration procedure described in Subsection 3.B.

\section{E. Stochastic Overlay Variations}

The final topic we want to discuss in this section is the uncertainty in the overlay measurement. Two distinct sources generating stochastic variations in the measured overlay signal are recognized. The first is the uncertainty coming from the DBO measurement itself and is extremely small, of the order of a few tenths of an angstrom. This very high measurement reproducibility was, in fact, the main reason why DBO was selected as the overlay methodology of choice for our application. It basically means that no significant contribution to the method error is expected from the DBO measurement. The second contribution is caused by small differences in the resist gratings used in the overlay measurement. These differences may, for example, originate from the complicated and random interaction between exposure radiation and light-sensitive molecules in the resist layer or any other stochastic process during exposure or processing of the wafers. The uncertainty introduced by these mechanisms is much larger than that of the DBO measurement and experiments have also shown that their magnitude may vary as a function of the PSG parameters.

Under these conditions, the ordinary least-squares minimization problem given in Eq. (21) is no longer optimal and should be replaced by the weighted least-squares minimization problem given by

$\tilde{\alpha}_{\text {min }}=\arg \min _{\alpha}\left\|Q\left(E \alpha-\left(\mathrm{OVL}_{\text {meas }}-\mathrm{OVL}_{\mathrm{ref}}\right)\right)\right\|^{2}$,

where $\mathrm{OVL}_{\text {ref }}$ denotes the calibration measurement on the reference system, which we assume to be independent of $\mathrm{OVL}_{\text {meas }}$, in which case $K$ simply becomes the sum of the experimentally determined covariance matrices

$$
K=K_{\text {meas }}+K_{\text {ref }},
$$

and $Q$ denotes the Cholesky decomposition of $K^{-1}$ according to

$$
K^{-1}=Q Q^{*} .
$$

The optimal generalized least-squares estimator, $\tilde{\alpha}_{\text {min }}$, for the Zernike coefficients describing the aberration state of the lithographic system, can then be explicitly written as

$$
\tilde{\alpha}_{\min }=\left(E^{*} K^{-1} E\right)^{-1} E^{*} K^{-1}\left(O V L_{\text {meas }}-O \mathrm{OV}_{\mathrm{ref}}\right),
$$

where we have now correctly accounted for the PSGtarget-specific overlay measurement uncertainty and the additional uncertainty introduced by the calibration measurement.

An additional uncertainty related problem that we are facing for the current measurement reticles is that the 420 targets included in the measurement modules do not sample the pupil optimally. As a result, the conditioning of the resulting linear system is not very good and this gives rise to a relatively large variance for specific (spherical) Zernikes. In this case, the least-squares estimation can be further improved by exploiting a Tikhonov-like regularization approach, which introduces an additional term in the cost function:

$$
\begin{aligned}
\tilde{\alpha}_{\min }(\zeta)= & \arg \min _{\alpha}\left\|Q\left(E \alpha-\left(\mathrm{OVL}_{\mathrm{meas}}-\mathrm{OVL}_{\mathrm{ref}}\right)\right)\right\|^{2} \\
& +\zeta^{2}\left\|D\left(\alpha-\alpha_{p}\right)\right\|^{2}
\end{aligned}
$$


Here $D$ is the regularization operator and $\alpha_{p}$ the prior mean, which are both set based on prior knowledge of typical aberration states of lithographic systems. The parameter $\zeta \geq 0$ determines the importance of the regularization term. Obtaining a good recipe for an optimal choice of $\zeta$ is a non-trivial task. For a more detailed description of the applied regularization techniques and the determination of the regularization parameter, the reader is referred to [7].

\section{Experimental Verification of the Method}

In this section we present the experimental validation of our method. We shall start by discussing the hardware used and we will describe the applied experimental procedures in detail, aimed at avoiding the experimental complications discussed in the previous section. Subsequently, aberration retrieval results are presented for the case that a known aberration was intentionally introduced to the projection lens of a lithographic system and, additionally, these wafer-based reconstruction results are compared to the aberrations measured by the on-tool aberration sensors.

\section{A. Reticle Design}

The most important components required by the PSG-OVL aberration metrology method are the two matched PSG and REF reticles required to construct the PSG-OVL targets. For the reticle set used in the experiments presented in this paper, the toplevel layout is identical. They contain $7 \times 7$ measurement modules, where each module consists of 420 PSG-based overlay targets. In turn, each overlay target consists of several grating stacks that together allow one to determine a single overlay value (see also Fig. 4). The grating stacks themselves are built up from two stacked $40 \mu \mathrm{m} \times 40 \mu \mathrm{m}$ gratings, where the top and bottom grating are constructed from the PSG and REF reticle, respectively. Basically, the only difference between the two reticle designs is the unit cell used to fill the $40 \mu \mathrm{m} \times 40 \mu \mathrm{m}$ grating areas. [Note that dimensions are given at wafer level (1X) and that dimensions at reticle level are 4 times larger.] For the PSG reticle, the unit cell definitions are given in Subsections 2.B and 2.E for the 1D- and 2D-PSG cases, respectively. The gratings used in the REF reticle design are simple LS gratings, which have the same effective pitch, $P$, and grating orientation, $\theta$, as the corresponding grating on the PSG reticle.

As mentioned above, the modules on the aberration measurement reticles contain 420 PSG-OVL targets. This set is composed from 28 different PSG-OVL targets (three 1D-PSGs and 25 2D-PSGs), all included at 15 different orientations (rotation angles in the reticle plane). For the current reticles, we have included three grating stacks per PSG-OVL target, and this results in a minimum area required for a complete module that is a little bit larger than $2 \mathrm{~mm}^{2}$. Note that on our test reticles the module size is substantially larger due to the inclusion of visually observable labels and additional test and calibration features. Nevertheless, our test reticles already include $7 \times 7$ modules, thus allowing one to also measure the field dependence of the aberrations in a single exposure.

\section{B. Experiment Description}

Experimental verification of the method is executed as follows. First, a set of reference wafers is created: using the complimentary binary REF reticle, coated silicon wafers are exposed and subsequently etched, stripped, and cleaned. The chosen illumination condition used to print the reference gratings was a wide conventional setting ( $0.94 \sigma$ at $1.35 \mathrm{NA})$, this in order to minimize the impact of possible aberrations on the reference features. The etch depth for the reference gratings is $30 \mathrm{~nm}$.

The chosen resist process for all our experiments is $105 \mathrm{~nm}$ JSR resist AIM5484 on top of $95 \mathrm{~nm}$ Brewer BARC ARC29SR; this should result in sufficient planarization of the etched reference gratings. All coatings and development are done inline on a Sokudo Duo track fitted to an ASML NXT:1950Ai scanner. Before starting experiments, we determined the smallest safe conventional illumination possible on the scanner $(0.122 \sigma$ at $1.35 \mathrm{NA})$. We then determined the dose at which all required phase gratings would print simultaneously to an acceptable pattern by qualitative SEM inspection. That exposure dose was then used for all experiments.

Each experiment consists of a set of exposures on the reference wafers using the above-mentioned resist process and tools. The use of so-called imagetuner subrecipes allowed us to dial in specific aberration offsets per wafer. The validity of each subrecipe was first verified by measuring the wavefront using the scanner's own integrated interferometer when applying the offsets requested in the subrecipe. For the exposures themselves, we used a specific scanner test that would also allow us to measure the wavefront before and after each exposure with the scanner's interferometer while still working inline with the track to bake and develop each wafer immediately after exposure.

Measurements were then performed on each wafer on a YieldStar S200 and raw pupil data was gathered that was subsequently processed offline to determine the overlay error for each PSG target. These measured overlay values, together with uncertainty information gathered from multiple measurements, then serve as input to the minimization problems defined in Eqs. (24) and (28), which, upon solving, provide us with estimates of the aberration state of the lithographic scanner at the time of the PSG reticle exposure.

\section{Experimental Results}

In this subsection we give an overview of the experimental work done to validate the method. We begin with an experiment in which a single non-zero Zernike $\left(Z_{7}=3 \mathrm{~nm}\right)$ has been dialed in as an 
aberration offset of the lithographic system. This intentionally detuned system is subsequently used to print the PSG reticle on top of the reference features already on the wafers. Next, the overlay is measured for all targets in a single module and, based on these measured overlay values, we try to recover the magnitude of the dialed-in Zernike term. In Fig. 16 the Zernike estimates obtained with the PSG-OVL method are shown. The bar plot on the top pertains to retrieval based on the raw measured overlay values, while the finite source is correctly accounted for according to Subsection 3.A. It may be observed that, although a $Z_{7}$ with a magnitude between 2 and $3 \mathrm{~nm}$ is detected, the method erroneously reconstructs large values for several other Zernikes, with most profound values for the spherical aberration terms $\left(Z_{16}, Z_{25}\right.$, and $\left.Z_{36}\right)$. These large spherical terms are mainly due to the PSG asymmetry effect discussed in Subsection 3.B. Therefore, by applying the calibration proposed in Subsection 3.B, a strong improvement can be achieved, resulting in the aberration retrieval result shown in the middle row of Fig. 16. Note that, due to this calibration step, the Zernikes shown in the middle row of Fig. 16, and in all subsequent figures, now represent the aberrations of the measured system relative to the reference system on which the calibration was based. Even for the calibrated result, some reconstructed spherical Zernikes remain substantial. A systematic analysis of this phenomenon has shown that it is caused by the suboptimal sampling of the aberration function achieved by the current set of measurement reticles. As a result, the conditioning of the linear system being solved is not optimal and, in this case, results in relatively large uncertainty, specifically for the spherical Zernikes. A proven concept to improve estimates from poorly conditioned systems is the use of regularization, and doing so further improves the Zernikes estimates obtained with the PSG-OVL method, as is shown in the bottom row of Fig. 16 . In the remainder of this subsection, all presented reconstruction results will be obtained with regularization and are based on calibrated overlay data.

The results of a second experiment, in which a cocktail of three non-zero Zernikes $\left(Z_{8}=2 \mathrm{~nm}\right.$, $Z_{10}=-2 \mathrm{~nm}$, and $Z_{27}=2 \mathrm{~nm}$ ) was dialed in, is shown in Fig. 17. Also in this experiment, a very good correlation is observed between the requested and retrieved Zernike terms and all residual Zernikes are smaller than $1 \mathrm{~nm}(1 \mathrm{~nm} \approx 5 \mathrm{~m} \lambda$ for a lithographic system operating at $193 \mathrm{~nm}$ ). This gives a good indication that the PSG-OVL method can determine arbitrary aberration states and does not suffer from a strong cross correlation between different aberration terms.

So far, reconstruction results are shown for only odd Zernike terms. As explained in Subsection 3.D, the aberration-induced overlay sensitivity of even Zernikes is reduced when the intended forbidden orders of a PSG are non-zero. The impact of this phenomenon may be observed in the retrieval results
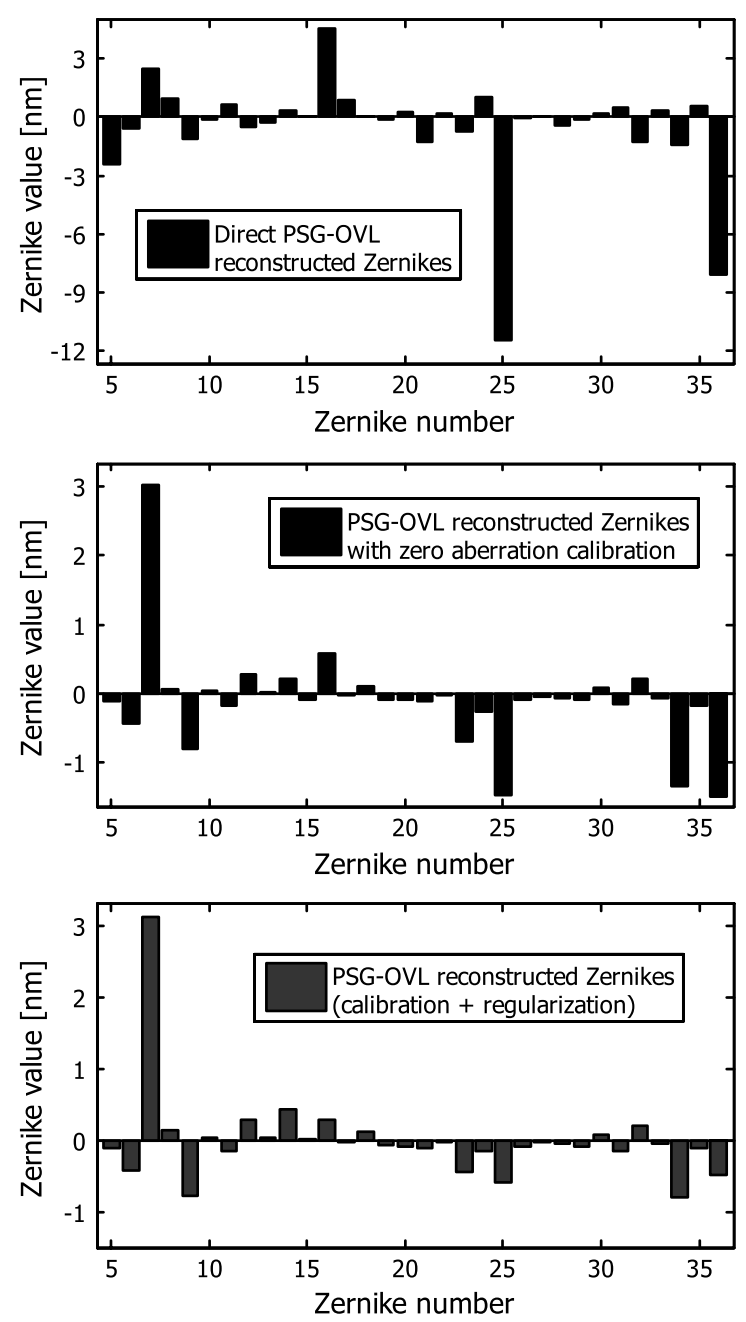

Fig. 16. Zernikes estimated with the PSG-OVL method for the case that a single non-zero coefficient $\left(Z_{7}=3 \mathrm{~nm}\right)$ was dialed in during exposure of the PSG layer onto the wafer. On the top, estimates for the Zernikes $\left(Z_{5}-Z_{36}\right)$, obtained from the raw overlay measurements, are shown. In the middle, the corresponding retrieval result is shown for the case that the measured overlay values are first calibrated with respect to an aberration-free reference system. The bottom row shows the calibrated result when additional regularization is applied in the fitting procedure.

shown in Fig. 18, where a cocktail of even Zernikes $\left(Z_{5}=2 \mathrm{~nm}, \quad \overline{Z_{13}}=-2 \mathrm{~nm}, \quad\right.$ and $\left.Z_{17}=2 \mathrm{~nm}\right)$ is dialed in during exposure. In the top row, the

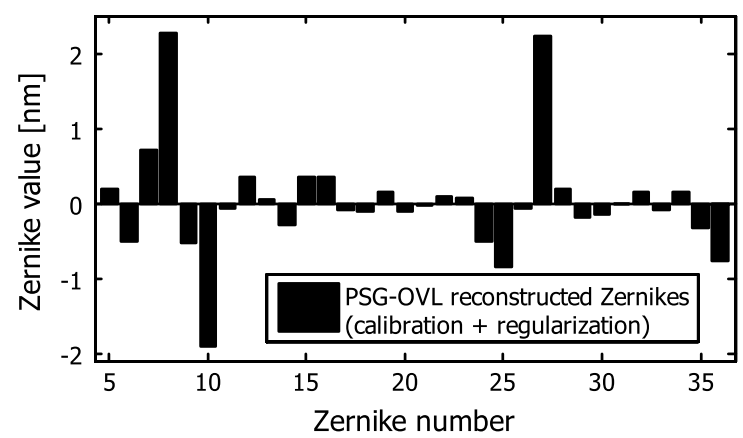

Fig. 17. PSG-OVL reconstructed Zernikes for a requested cocktail of three non-zero coefficients $\left(Z_{8}=2 \mathrm{~nm}, Z_{10}=-2 \mathrm{~nm}\right.$, and $Z_{27}=2 \mathrm{~nm}$ ). 

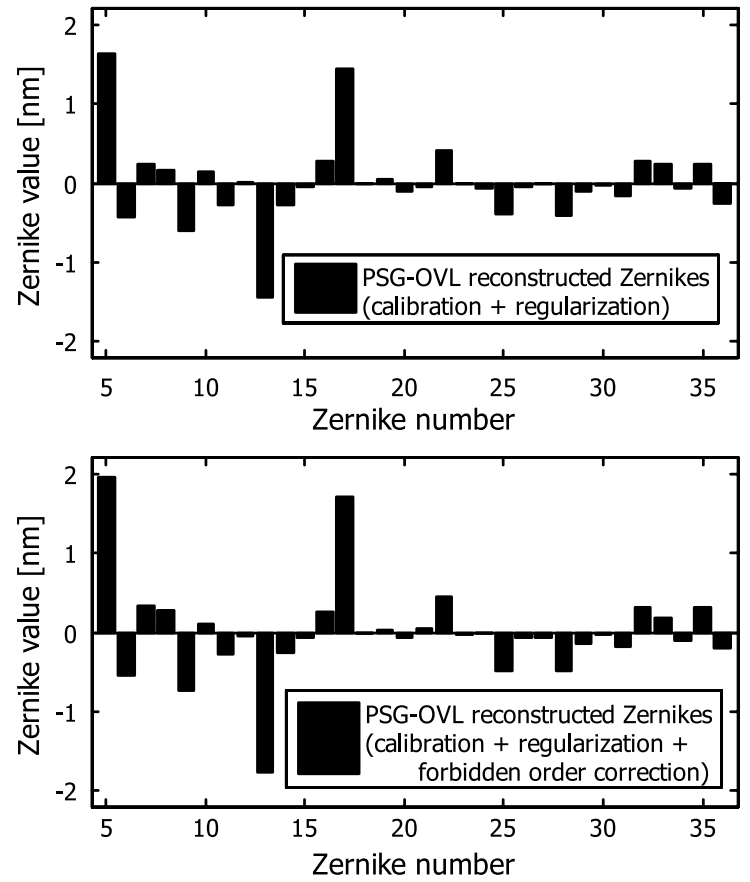

Fig. 18. Zernikes estimated with the PSG-OVL method for the case that a cocktail of three even Zernikes $\left(Z_{5}=2 \mathrm{~nm}\right.$, $Z_{13}=-2 \mathrm{~nm}$, and $Z_{17}=2 \mathrm{~nm}$ ) is dialed in. The top axis shows the Zernike estimates obtained using both calibration and regularization. The results in the bottom panel are obtained using an additional correction based on the AIMS-measured residual forbidden-order intensities generated by the PSG reticle.

PSG-OVL-based estimates are shown when this effect is neglected, indeed showing Zernike estimates that are too small. A significant improvement is observed when this effect is accounted for in the model using AIMS-measured forbidden-order information (see bottom row Fig. 18). Note that AIMS measurements were available only for a subset of all PSGs and that missing data was supplemented by extrapolated values. Therefore, the improvement achieved by this correction is expected to be even more drastic when forbidden-order information is available for all PSGs used in the reconstruction.

Finally, we present in Fig. 19 a comparison between the aberration coefficients measured with the on-tool aberration sensor of the exposure system

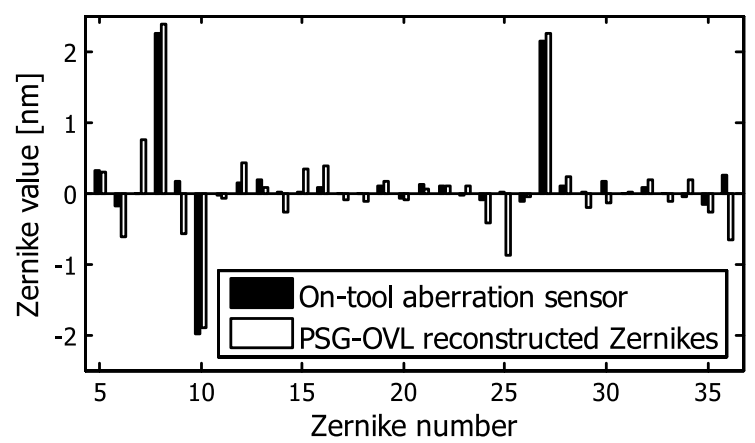

Fig. 19. Comparison between the aberration coefficients obtained with the on-tool aberration sensors of the exposure tool and the PSG-OVL method (identical experimental settings as in Fig. 17). and those estimated by the PSG-OVL method. Also here, a very good agreement between both measurements is observed for the imposed non-zero Zernike coefficients. A comparison of the residuals of both methods shows larger differences between the two methods, where it should be pointed out that the residuals of the PSG-OVL method are slightly larger than those of the on-tool sensor. Nevertheless, it is remarkable that such a good agreement, down to the subnanometer regime, can be achieved between a wafer-based method and a dedicated on-tool sensor that is based on shearing interferometry.

\section{Conclusions and Discussion}

In this paper we have presented a new methodology for deriving the aberration state of a lithographic projection system based on wafer metrology data. This method uses the aberration-induced image shift of specially designed overlay targets as a measurand based on which the phase aberration function in the exit pupil of the lithographic system can be reconstructed. In Section 2 it was explained how PSG targets can be designed such that a very simple relation emerges between their relative image position and the difference of the phase aberration function evaluated at the location of their diffraction orders. Moreover, it was shown that, using a combination of 1D- and 2D-PSGs, the phase difference between different points throughout the pupil can be probed, eventually providing enough information to accurately estimate the Zernikes representing the phase aberration function. At the end of Section 2, this methodology was proven to be feasible in simulation via a numerical experiment based on a lithographic simulator and showed an aberration reconstruction accuracy of better than $5 \mathrm{~m} \lambda$.

Before continuing with the experimental verification of the method, first a number of practical complications were addressed in Section $\underline{3}$. There it was shown that, for all complications currently recognized, their impact can either be accounted for in the PSG-OVL model or their negative effect can be strongly reduced through a calibration step. This was illustrated in Section 4 through a number of experiments, with and without the mentioned corrections, which showed the most drastic improvement when the reference scanner calibration was included. Altogether, using all model refinement and corrections discussed in Section $\underline{3}$, the accuracy of the wafer-based aberration estimation method introduced in this paper was shown to be remarkably good. The retrieval error, with respect to the dialed-in aberration and independent measurement with the on-tool aberration sensors, is below $1 \mathrm{~nm}$, which corresponds to approximately $5 \mathrm{~m} \lambda$ at the exposure wavelength of the lithographic system being evaluated.

Nonetheless, we still observe residuals in the reconstructed Zernikes that are between 0.5 and $1 \mathrm{~nm}$. Although extremely small, aberrations of this order of magnitude are nowadays considered 
significant by the lithographic industry. Therefore, the aim of the current research was to achieve an aberration estimation accuracy better than $0.5 \mathrm{~nm}$ and, in this respect, the current work still requires additional effort to reach the desired accuracy. Fortunately, we do see several opportunities to further improve and enhance the presented method and we shall discuss those here briefly.

For the experiments presented in this paper, we have used a set of reticles that was designed based on our initial understanding and ideas regarding wafer-based aberration metrology. Since that time, we have gained much more insight on the physical processes and error sources relevant for our method. Basically, we now know that our reticles are, in many ways, not optimal for the aimed aberration metrology application. For example, the current set of 420 PSG targets does not sample the pupil in an optimal way and this leads to poor conditioning and significant cross correlation in the linear system being solved to estimate Zernikes. In fact, this is the main reason why regularization does improve the aberration reconstruction results obtained with the current reticles. Apart from selecting the optimal PSG targets, there is also still room to optimize the targets themselves. It was discussed in Subsection 3.D that, although the PSG designs are optimized to compensate mask $3 \mathrm{D}$ effects, there will always remain differences between the design and realized features, leading to non-zero forbidden diffraction orders. An experimental optimization approach, in which PSGs having slightly different design parameters are evaluated in terms of their measured forbiddenorder strength, could be a way to compensate these manufacturing defects, which are hard to parameterize.

Parallel to improving the PSG set and the individual designs, also the analysis and corrections presented in this paper can be further improved. For example, the forbidden-order correction mentioned in Subsection 3.D was based on AIMS measurements on just a subset off all PSGs, with the missing data being filled in by extrapolated values. Also, determining the target specific variance for the overlay via a large number of experiments, which then serves as input to the generalized least-squares problem, will contribute to an improved stability of the PSG-OVL method.

Altogether, we are convinced that, with a set of new optimized measurement reticles, complete reticle metrology data and accurate knowledge of the stochastic overlay behavior of the PSGs, the aimed aberration reconstruction accuracy for the method presented in this paper is well within reach. Consequently, we consider this method an excellent candidate to study dynamic aberration effects occurring during exposure of a wafer.

Appendix A: Conversion between $Z_{t}$ and $Z_{n}^{m}$

In this appendix we provide a conversion rule to go from a Zernike function in single-index notation, $Z_{t}$,
(FRINGE convention) to a Zernike function in double-index notation, $Z_{n}^{m}$, and vice versa.

a. Conversion: $Z_{t} \rightarrow Z_{n}^{m}$

For $t=1,2, \cdots$, we let

$$
q=\lfloor\sqrt{t-1}\rfloor, \quad p=\left\lfloor\frac{t-q^{2}-1}{2}\right\rfloor,
$$

and

$$
n=q+p, \quad m=q-p ;
$$

then a single-index Zernike function, $Z_{t}(\rho, \theta)$ equals

$$
\begin{aligned}
Z_{t}(\rho, \theta)= & R_{n}^{m}(\rho)\left\{\left(t-q^{2}-2 p\right) \cos m \theta\right. \\
& \left.+\left[1-\left(t-q^{2}-2 p\right)\right] \sin m \theta\right\} \\
= & Z_{n}^{m}(\rho, \theta) .
\end{aligned}
$$

b. Conversion: $Z_{n}^{m} \rightarrow Z_{t}$

When $n, m$ are positive integers such that $n-m$ is even and non-negative, then

$$
\begin{aligned}
& R_{n}^{m}(\rho) \cos m \theta=Z_{\left(\frac{n+m}{2}\right)^{2}+n-m+1}, \\
& R_{n}^{m}(\rho) \sin m \theta=Z_{\left(\frac{n+m}{2}\right)^{2}+n-m+2},
\end{aligned}
$$

with Eq. (A5) only valid for $m \neq 0$.

\section{Appendix B: 2D-PSG Unit Cell Definition}

In this appendix we discuss the relation between the 2D-PSG unit cell definition, its corresponding pupil positions of its allowed diffraction orders, and the parameters we use to define them.

We use four parameters that completely define the 2D-PSG targets used by our method: the pitch, $P$, of the resulting $1 \mathrm{D}$ pattern generated from the interference between the two orders close the pupil edge, the distance $\rho_{1}$ between the pupil center and the allowed diffraction order closest to the pupil edge, the fraction $\xi$ being the ratio between $\rho_{3}$ and $\rho_{0}$ that are defined in Fig. 20, and, finally, the grating orientation $\phi$. All distances defined in Fig. $\underline{20}$ are then given by

$$
\begin{aligned}
& \rho_{0}=\frac{\lambda}{\mathrm{NA} P}, \\
& \rho_{1}=\rho_{1}, \\
& \rho_{2}=\sqrt{(1-\xi)^{2} \rho_{0}^{2}+\rho_{1}^{2}-\xi^{2} \rho_{0}^{2}}, \\
& \rho_{3}=\xi \rho_{0}, \\
& \rho_{A}=(1-\xi) \rho_{0}
\end{aligned}
$$




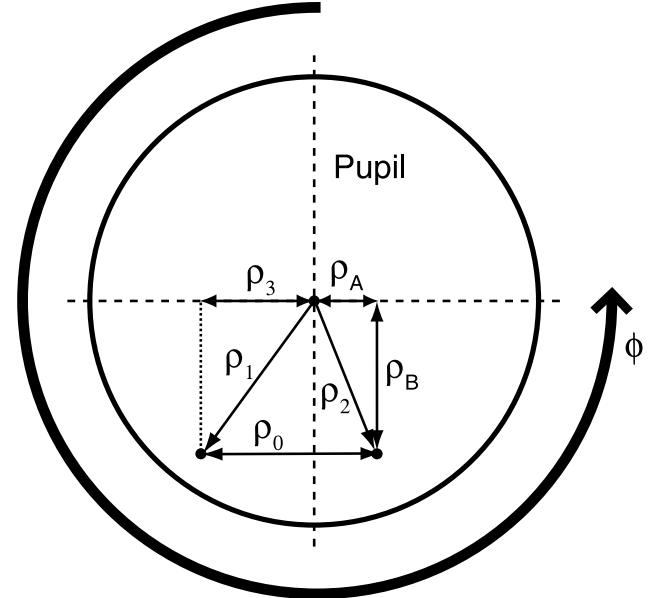

Fig. 20. Definition of the relevant distances in reciprocal (pupil) space.

$$
\left.\rho_{B}=\sqrt{(} \rho_{1}^{2}-\xi^{2} \rho_{0}^{2}\right),
$$

where $\lambda$ and NA are again the wavelength and NA of the lithographic system.

Next, we can also express the real-space parameters defined in Fig. 21 in terms of the 2D-PSG parameters and the distances defined in Fig. 20:

$$
\begin{gathered}
\omega=\arccos \left[\frac{\rho_{1}^{2}+\rho_{2}^{2}-\rho_{0}^{2}}{2 \rho_{1} \rho_{2}}\right], \\
\Psi=\arccos \left[\frac{\rho_{0}^{2}+\left(2 \sin \left(\frac{\omega}{2}\right) \rho_{2}\right)^{2}-\left(\rho_{1}-\rho_{2}\right)^{2}}{4 \sin \left(\frac{\omega}{2}\right) \rho_{0} \rho_{2}}\right] \\
A_{\min }=\frac{\lambda}{2 \mathrm{NA} \rho_{1}} \sqrt{\left(\frac{1}{\cos \left(\frac{\omega}{2}\right)}\right)^{2}+\left(\frac{1}{\sin \left(\frac{\omega}{2}\right)}\right)^{2}},
\end{gathered}
$$

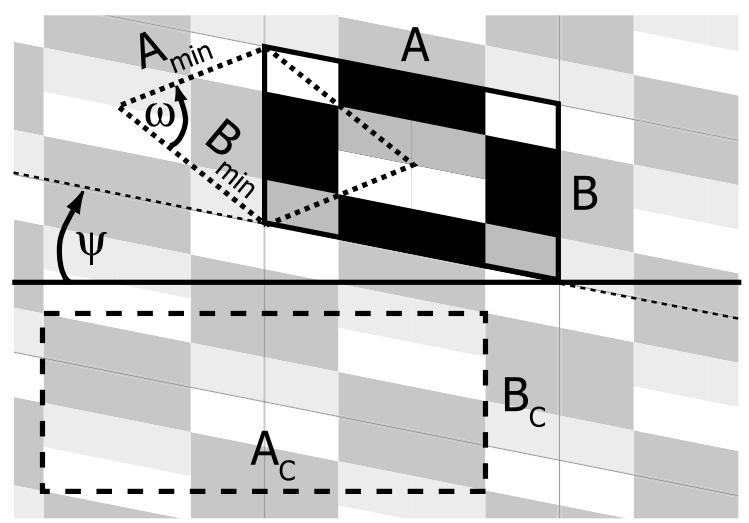

Fig. 21. Definition of the real-space variables and relation between the minimum unit cell (dotted box), computational unit cell (dashed box) and the unit cell as defined in Fig. 7 .

$$
\begin{gathered}
B_{\min }=\frac{\rho_{1}}{\rho_{2}} A_{\min }, \\
A_{C}=\frac{\Xi \lambda}{\mathrm{NA} \xi \rho_{0}}, \\
B_{C}=\frac{\lambda}{\mathrm{NA} \sqrt{\rho_{1}^{2}-\xi^{2} \rho_{0}^{2}}},
\end{gathered}
$$

where $\xi$ is a rational number between $(1 / 2) \leq \xi \leq 1$, and $\Xi$ is the numerator of the smallest possible rational representation of $\left(\begin{array}{c}\xi \\ 1-\xi\end{array}\right)$. Note that the angles $\omega$ and $\Psi$, together with the minimum unit cell rib lengths, are sufficient to define a 2D-PSG in a reticle design. However, the super cell defined by $A_{C}$ and $B_{C}$ is also provided because lithographic simulators commonly require such a rectangular (Manhattentype) unit cell to simulate the proposed 2D-PSGs.

For a given 2D-PSG design to be applicable, it should satisfy requirements similar to those in the 1D-PSG case; nonforbidden higher orders should remain outside the pupil and the effective pitch of the grating image should be observable by the DBO tool. For a 2D-PSG, this translates into the following constraints:

$$
\begin{gathered}
\frac{2 \lambda}{\mathrm{NA}(1+\sigma)} \leq P \leq \frac{\lambda_{\mathrm{DBO}}}{C \mathrm{NA}_{\mathrm{DBO}}}, \\
\sqrt{(1+\sigma)^{2}-(4-4 \xi)\left(\frac{\lambda}{\mathrm{NA} P}\right)^{2}} \leq \rho_{1} \leq 1, \\
1-\frac{\left((1+\sigma)^{2}-\rho_{1}^{2}\right)(\mathrm{NA} P)^{2}}{4 \lambda^{2}} \geq \xi \geq \frac{1}{2} .
\end{gathered}
$$

\section{Appendix C: Semianalytic Computation of Phase Difference Averages}

After linearizing the exponential comprising the phase difference, we must evaluate the average of the phase difference over a specific subset of the reference pupil. This subset consists of the intersection region of three disks, namely, the pupil disk, a displaced copy of the pupil disk, and the supporting disk of the scanner's illumination monopole. For the sake of mathematical convenience we will use in this appendix the double-index complex exponential representation of the Zernike functions, and we assume that the disk pertaining to the scanner monopole has radius 1 and center $o$, while the two pupil disks have radius $r$ and centers $\underline{\nu}_{1}$ and $\underline{\nu}_{2}$, respectively. See Fig. 22 for the configuration, in which the hatched region $S$ is the averaging region. 


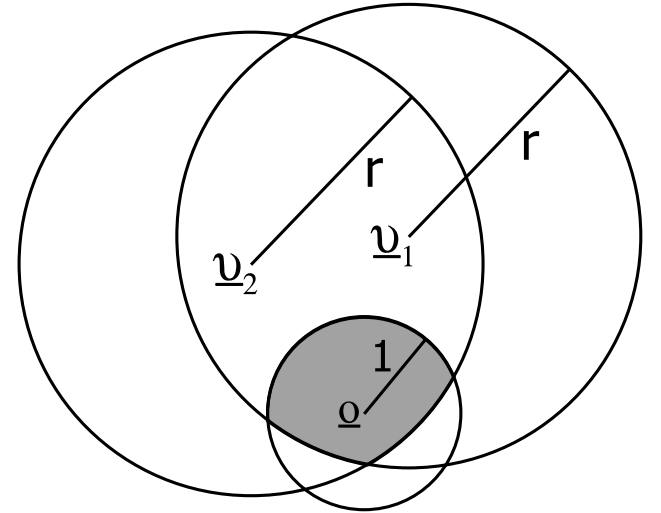

Fig. 22. Integration range $S$ in Eq. (C1) consisting of the intersection of two pupil disks centered at $\underline{\nu}_{1}$ and $\underline{\nu}_{2}$ and a third disk centered at $\underline{o}$ defined by the scanner's illumination monopole, with choice of origin and radii facilitating solving the mathematical problem.

The mathematical problem at hand thus consists of solving the integrals

$$
\iint_{S} Z_{n^{\prime}}^{m^{\prime}}\left(\frac{\underline{\nu}-\underline{\nu}_{1,2}}{r}\right) \mathrm{d} \underline{\nu}
$$

where $Z_{n^{\prime}}^{m^{\prime}}$ denotes the complex Zernike circle polynomial vanishing outside the unit disk, given as

$$
\begin{array}{r}
Z_{n^{\prime}}^{m^{\prime}}(\underline{\nu}) \equiv Z_{n^{\prime}}^{m^{\prime}}\left(\rho e^{i \theta}\right)=R_{n^{\prime}}^{\left|m^{\prime}\right|}(\rho) e^{i m^{\prime} \theta}, \\
0 \leq \rho \leq 1, \quad 0 \leq \theta \leq 2 \pi,
\end{array}
$$

for integer $n^{\prime}, m^{\prime}$ such that $n^{\prime}-\left|m^{\prime}\right|$ is even and nonnegative. The evaluation of Eq. (C1) can be done using the approach in []. Accordingly, focusing on the case $\underline{\nu}_{1}$, we have

$$
\begin{aligned}
& \iint_{S} Z_{n^{\prime}}^{m^{\prime}}\left(\frac{\underline{\nu}-\underline{\nu}_{1}}{r}\right) \mathrm{d} \underline{\nu} \\
& \quad=\iint_{\underline{\nu} \leq 1} Z_{n^{\prime}}^{m^{\prime}}\left(\frac{\underline{\nu}-\underline{\nu}_{1}}{r}\right) Z_{0}^{0}(\underline{\nu})\left(Z_{0}^{0}\left(\frac{\underline{\nu}-\underline{\nu}_{2}}{r}\right) Z_{0}^{0}(\underline{\nu})\right)^{*} \mathrm{~d} \underline{\nu} \\
& \quad=\sum_{n, m} \frac{\pi}{n+1} \beta_{n, 1}^{m}\left(\beta_{n, 2}^{m}\right)^{*},
\end{aligned}
$$

with $\beta_{n, 1}^{m}$ and $\beta_{n, 2}^{m}$ the Zernike coefficients with respect to the unit disk of $Z_{n^{\prime}}^{m^{\prime}}\left(\frac{\underline{\nu}-\underline{\underline{\nu}}}{r}\right) Z_{0}^{0}(\underline{\nu})$ and $Z_{0}^{0}\left(\frac{\underline{\nu}-\underline{\nu}_{2}}{r}\right) Z_{0}^{0}(\underline{\nu})$, respectively. These $\beta$ can be cast into the form of a correlation integral, as in [8], Eq. (8):

$$
\begin{gathered}
\beta_{n, 1}^{m}=\frac{n+1}{\pi} \iint Z_{n^{\prime}}^{m^{\prime}}\left(\frac{\underline{\underline{\nu}}-\underline{\nu}_{1}}{r}\right)\left(Z_{n}^{m}(-\underline{\nu})\right)^{*} \mathrm{~d} \underline{\nu}, \\
\beta_{n, 2}^{m}=\frac{n+1}{\pi} \iint Z_{0}^{0}\left(\frac{\underline{\nu}-\underline{\nu}_{2}}{r}\right)\left(Z_{n}^{m}(-\underline{\nu})\right)^{*} \mathrm{~d} \underline{\nu},
\end{gathered}
$$

and these correlation integrals have been computed in [8], Eq. (9) and Theorem 2.1. Thus (choosing $c^{\prime}=r$, $c=1,\left(\tau^{\prime}, \eta^{\prime}\right)=-\underline{\nu}_{1},(\tau, \eta)=(\underline{o})$,

$$
\beta_{n, 1}^{m}=\frac{n+1}{\pi} \sum_{n^{\prime \prime}} C_{n^{\prime} n, n^{\prime \prime}}^{m^{\prime} m} Z_{n^{\prime \prime}}^{m^{\prime}-m}\left(-\frac{\underline{\nu}_{1}}{r+1}\right), \quad\left|\underline{\nu}_{1}\right| \leq r+1,
$$

and a similar formula for $\beta_{n, 2}^{m}$. The summation in Eq. ( $\underline{\mathrm{C} 6})$ is over all integers $n^{\prime \prime}$ such that $n^{\prime \prime}-\left(n^{\prime}+n\right)$ is even and non-negative. The $C$ are given as

$$
\begin{aligned}
C_{n^{\prime} n, n^{\prime \prime}}^{m}= & \left(\frac{r}{r+1}\right)^{2} \frac{(-1)^{n}\left(n^{\prime \prime}+1\right) \pi}{\left(n^{\prime}+1\right)(n+1)} \\
& \times\left[S_{n^{\prime} n}^{n^{\prime \prime}+1}-S_{n^{\prime}+2, n}^{n^{\prime \prime}+1}-S_{n^{\prime}, n+2}^{n^{\prime \prime}+1}+S_{n^{\prime}+2, n+2}^{n^{\prime \prime}+1}\right],
\end{aligned}
$$

with

$$
\begin{aligned}
S_{i j}^{k+1}= & \frac{\left(\frac{1}{2}(k+i+j)\right) !\left(\frac{1}{2}(k-i-j)\right) !}{\left(\frac{1}{2}(k+i-j)\right) !\left(\frac{1}{2}(k-i+j)\right) !} \\
& \times \frac{r^{i}}{(r+1)^{i+j}}\left(P_{\frac{1}{2}(k-i-j)}^{(i, j)}\left(\frac{1-r}{1+r}\right)\right)^{2},
\end{aligned}
$$

for integers $i, j, k \geq 0$ such that $k-i-j$ is nonnegative and even and $S_{i, j}^{k+1}=0$ otherwise. The $P_{l}^{(\alpha, \beta)}(x)$ are Jacobi polynomials; see [9], Chapter 22.

The series in Eqs. (C3) and (C6) are rather slowly convergent, and so the computations must be done efficiently. The $S_{i j}^{k+1}$ can be expressed in terms of the generalized Zernike functions of [10] according to

$$
\frac{r^{i}}{(r+1)^{i+j}}\left(P_{\frac{1}{2}(k-i-j)}^{(i, j)}\left(\frac{1-r}{1+r}\right)\right)^{2}=\left(1-\rho^{2}\right)^{-\frac{i}{2}} R_{k-i}^{j, i}(\rho),
$$

with $\rho=(1+r)^{-1 / 2} \in(0,1)$. Finally, according to [10], Theorem 5.2 (correcting two minor typos), the generalized Zernike functions can be computed in a DFT format as follows. Let $n$ and $m$ be integers such that $n-|m|$ is even and non-negative, and let $q=(1 / 2)(n+|m|)$. Furthermore, let $\alpha>-1$ and denote by $C_{n}^{\alpha+1}$ the Gegenbauer polynomial of [9], Chapter 22. Then for any integer $N$ such that $N>n+|m|$, we have

$$
\begin{aligned}
& \left(\begin{array}{c}
q+\alpha \\
q
\end{array}\right)\left(1-\rho^{2}\right)^{-\alpha} R_{n}^{|m|, \alpha}(\rho) \\
& \quad=\frac{1}{N} \sum_{k=0}^{N-1} C_{n}^{\alpha+1}\left(\rho \cos \frac{2 \pi k}{N}\right) e^{-2 \pi i \frac{k m}{N}}
\end{aligned}
$$

The prefactors

$$
a_{k}=\frac{\left(\frac{1}{2}(k+i+j)\right) !\left(\frac{1}{2}(k-i-j)\right) !}{\left(\frac{1}{2}(k+i-j)\right) !\left(\frac{1}{2}(k-i+j)\right) !}
$$

in Eq. (C8) should be computed for all $k$ such that $k-i-j \overline{\text { is }}$ even and non-negative. We can do that recursively according to 


$$
a_{k=i+j}=\left(\begin{array}{c}
i+j \\
i
\end{array}\right)
$$

and, for $k=i+j, i+j+2, \cdots$,

$$
a_{k+2}=\frac{\left(\frac{1}{2}(k+i+j)+1\right)\left(\frac{1}{2}(k-i-j)+1\right)}{\left(\frac{1}{2}(k+i-j)+1\right)\left(\frac{1}{2}(k-i+j)+1\right)} a_{k}
$$

The authors would like to acknowledge Brid Connolly, Martin Sczyrba, and their team (from Toppan Photomask, Inc. in Dresden) for their excellent support and input during realization and optimization of the nonstandard reticles required by our method. In addition, we would like to thank our ASML coworkers Twan Schellekens, Joep de Vocht, Fahong Li, Ton Kiers, Jan Baselmans, Nico Vanroose, Maxim Pisarenco, Patrick Tinnemans, and Jan Mulkens, who all contributed in their own way to this work.

\section{References}

1. H. Nomura, "New phase-shift gratings for measuring aberrations," Proc. SPIE 4346, 25-35 (2001).

2. M. A. van de Kerkhof, W. de Boeij, H. Kok, M. Silova, J. Baselmans, and M. Hemerik, "Full optical column characterization of DUV lithographic projection tools," Proc. SPIE 5377, 1960-1970 (2004).

3. C. Kittel, Introduction to Solid State Physics, 5th ed. (Wiley, 1976).

4. P. Vanoppen and T. Theeuwes, "Lithographic scanner stability improvements through advanced metrology and control," Proc. SPIE 7640, 764010 (2010).

5. M. Ebert, H. Cramer, W. Tel, M. Kubis, and H. Megens, "Combined overlay, focus and CD metrology for leading edge lithography," Proc. SPIE 7973, 797311 (2011).

6. T. Hastie, R. Tibshirani, and J. J. H. Friedman, The Elements of Statistical Learning (Springer, 2001), Vol. 1.

7. M. Pisarenco and I. Setija, "Compact discrepancy and chisquared principles for over-determined inverse problems" submitted to Inverse Methods.

8. A. J. E. M. Janssen, "Computation of Hopkins' 3-circle integrals using Zernike expansions," J. Eur. Opt. Soc. Rapid Pub. 6, 11059 (2011).

9. M. Abramowitz and I. A. Stegun, Handbook of Mathematical Functions (Dover, 1972).

10. A. J. E. M. Janssen, "A generalization of the Zernike circle polynomials for forward and inverse problems in diffraction theory," arXiv:1110.2369v1 (2011). 